\title{
Justifying Inference to the Best Explanation as a Practical Meta-Syllogism on Dialectical Structures
}

\author{
Gregor Betz*
}

October 1, 2011

\begin{abstract}
This article discusses how inference to the best explanation (IBE) can be justified as a practical meta-argument. It is, firstly, justified as a practical argument insofar as accepting the best explanation as true can be shown to further a specific aim. And because this aim is a discursive one which proponents can rationally pursue in - and relative to - a complex controversy, namely maximising the robustness of one's position, IBE can be conceived, secondly, as a meta-argument. My analysis thus bears a certain analogy to Sellars' wellknown justification of inductive reasoning [Sellars, 1969]; it is based on recently developed theories of complex argumentation [Betz, 2010a, 2011].
\end{abstract}

\section{Introduction}

Inference to the best explanation (IBE) has puzzled generations of philosophers and logicians. For, at first glance, IBEs appear to be straightforwardly fallacious. Take, for example, the famous teleological proof for the existence of God. This argument can be understood as an inference to the best explanation: We observe that all subdivisions of nature are closely adjusted. The existence of God, an intelligent being that created the universe, is presumably the best explanation of this observation. Therefore, God exists. Yet, a simple reconstruction yields,

(A1) All subdivisions of nature are adjusted to each other such that means are adapted to appropriate ends. (Explanandum $E$ )

(A2) The existence of God, an intelligent being that created the universe, implies that all subdivisions of nature are adjusted to each other such that means are adapted to appropriate ends. (Since that hypothesis $H$ explains $E$.)

(A3) Thus: God exists. (Explanans $H$ )

By deductive standards, this argument is clearly invalid. Inference to the best explanation seems to be, as has been widely acknowledged [e.g. Thagard, 1988,

*Karlsruhe Institute of Technology, Germany, email: gregor.betz@kit.edu. 
p. 140], a peculiar kind of the fallacy of affirming the consequence. However, this sort of inference appears to be both omnipresent and reliable - not only in everyday contexts, but in science, too. ${ }^{1}$

Let us have a brief look at premiss (A2). The reconstruction assumes that the explanatory hypothesis $H$ logically entails the explanandum $E$ (possibly by making use of some background assumptions which have been omitted). Such explanations which can be reconstructed as valid arguments shall be called "deductive." Accordingly, causal explanations (at least deterministic ones) and explanations that satisfy the deductive-nomological scheme represent examples of deductive explanations. (Yet, it should be noted upfront that this paper's analysis by no means implies that every argument satisfying the D-N scheme, let alone every deductive argument, represents an explanation.) The overall aim of this paper is to justify inference to the best deductive explanation (IBdE), though I shall briefly comment on statistical explanations in the concluding section. ${ }^{2}$

The general reason why IBdE seems to be fallacious is that, in deductive reasoning, a statement $A$ being a sufficient warrant for a statement $B$ does not entail that $B$ is a sufficient warrant for $A$. We have here an asymmetry, and this asymmetry makes IBdE a puzzle. If IBdE were, however, to be analysed in a Bayesian framework [cf. Howson and Urbach, 2005], that puzzle would disappear. According to Bayesian Confirmation Theory, $A$ confirms $B$ iff the conditional degree of belief in $B$ given $A, P(B \mid A)$, is greater than the unconditional degree of belief in $B, P(B)$, i.e. iff $P(B \mid A) / P(B)>1$. Now, a simple theorem of probability theory states that

$$
P(B \mid A) / P(B)=P(A \mid B) / P(A)
$$

As a consequence, $B$ confirms $A$ if and only if $A$ confirms $B$. Provided that a deductive explanation $H$ always confirms the explanandum $E$ (as we have $P(E \mid H)=$ 1, taking all auxiliary assumptions for granted), the explanandum $E$ itself confirms the explanans $H$. Hence, Bayesian Confirmation Theory - instead of representing a threat to IBE, as van Fraassen [1989, pp. 160ff.] has argued — may help to justify it. A full-fledged justification of IBdE in Bayesian terms would have to show that for the best explanation $H^{*}$ :

$$
P\left(E \mid H^{*}\right) \geq P\left(E \mid H_{i}\right) \text { and } P\left(H^{*}\right) \geq P\left(H_{i}\right)
$$

\footnotetext{
${ }^{1}$ Note that inference to explanatory principles had already been at the heart of Aristotle's philosophy of science [cf. Losee, 2001, chapter 1].

${ }^{2}$ The focus on deductive explanations narrows - without, however, entirely removing - the contrast between IBE and the hypothetico-deductive account of confirmation. But unlike hypotheticodeductivism, the analysis carried out in this paper does not presuppose that some evidence $E$ confirms a hypothesis $H$ if and only if $H$ entails $E$.
} 
for all alternative explanations $H_{i} \cdot{ }^{3}$ As Hitchcock [2007] points out, that can only be accomplished in a piecemeal fashion: It has to be shown that whatever feature makes a hypothesis a "lovely" explanation [in the sense of Lipton, 2004] increases, at the same time, its prior probability or the likelihood of the explanandum given the hypothesis. This is how, within a Bayesian framework, it might be shown that the loveliest explanation is also the likeliest. ${ }^{4}$

At first glance, this paper appears to pursue quite different a strategy for justifying IBdE. First and foremost, we don't analyse IBdE in Bayesian terms. As a consequence, the suggested justification of IBdE does not buy into the assumptions and problems of Bayesian Confirmation Theory [cf. Earman, 1992, Mayo, 1996]. Whereas the general approach of this paper diverges significantly from a Bayesian analysis, the detailed argumentation, however, will reveal close analogies to the Bayesian reasoning I have just sketched, as will become more apparent as the argument unfolds.

The main idea of this paper can be put as follows. Accepting the loveliest explanation (i.e. the one which is superior in terms of simplicity, unificatory power, explanatory strength, etc.) as true is the best way of achieving certain intrinsic ${ }^{5}$, epistemic aims. Thus, to the extent that these aims should be realised, it is reasonable to consider the loveliest explanation as true. Accordingly, what underlies IBdE is in fact a practical syllogism. Since both the aim as well as the appropriate means figuring in that practical argument are evaluated with regard to our epistemic situation, this argument can be aptly characterised as a meta-syllogism. In order to elaborate this sketch, we have to understand more precisely (i) what exactly is the epistemic aim which one attempts to further by accepting the best explanation as true, (ii) why is it reasonable to pursue that aim in the first place, and (iii) why does accepting the best explanation promote that goal. These questions make up the overall agenda of this paper.

\footnotetext{
${ }^{3}$ Okasha [2000] claims that the loveliest explanation merely had to satisfy either $P\left(E \mid H^{*}\right) \geq$ $P\left(E \mid H_{i}\right)$ or $P\left(H^{*}\right) \geq P\left(H_{i}\right)$. But this is clearly insufficient for being the likeliest explanation since a high likelihood might very well be offset by a low prior probability and vice versa.

${ }^{4}$ Still, Schupbach and Sprenger [2011] provide an alternative Bayesian analysis: They define a probabilistic measure of a hypothesis' explanatory power $\mathcal{E}$ and prove that $\mathcal{E}$ is unique with a view to a set of conditions that spell out explanatory strength. The measure defined by Schupbach and Sprenger, however, is independent of the hypothesis' prior probability. That's why I am doubtful that $\mathcal{E}$ may yield a full explication of inference to the best explanation (since higher prior probability makes a hypothesis more lovely an explanation, or so it seems). Yet, Schupbach [2011, pp. 117-126] shows by computer simulation that the measure of explanatory power, $\mathcal{E}$, is nonetheless a valuable heuristics which approximates probabilistic, Bayesian, reasoning. I take it that these results stand in no contradiction with the argumentation unfolded in this paper. With a view to future investigation, it might be worthwhile to apply and study the measure of explanatory power by Schupbach and Sprenger in a dialectic framework and with a view to degrees of justification instead of degrees of belief.

${ }^{5}$ The rationale for an intrinsic aim does not depend on other aims. In particular, those aims with respect to which IBdE will be justified have to be established independently of our explanatory goals, since otherwise the justification of IBdE would obviously be trivial.
} 
In detail, the reasoning takes off with a brief account of Sellars' analysis of probability arguments in section 2. Sellars' interpretation of inductive reasoning, it is shown, bears a close analogy to the approach of this paper and may therefore be considered a blueprint for our own analysis. Section 3 paves the way for justifying IBdE by setting up the framework within which our analysis will be carried out, i.e. it provides a concise description of the theory of dialectical structures. On this background, the notion of a robust position a proponent can adopt in a controversial argumentation is introduced, as a technical term, in section 4. It is argued, moreover, that maximising the robustness of one's position may be considered a reasonable epistemic aim. Thus, section 4 directly addresses questions (i) and (ii) stated in the previous paragraph. Explanations can be reconstructed as arguments that make up a complex argumentation, as section 5 shows. This allows us to analyse alternative explanations coherently in terms of the theory of dialectical structures. Since explanatory qualities that make an explanation a particularly lovely one are reflected in specific properties of the corresponding dialectical structure, section 6 succeeds in demonstrating that accepting the best explanation as true maximises the robustness of the accordingly extended position. This is what question (iii) called for. Having justified IBdE as a practical meta-argument, we will, in section 7, apply our account of IBdE to analyse three prominent examples of abductive inference: Semmelweis's explanation of childbed fever, Meselson's and Stahl's investigation into DNA replication, and the discussion of the theory of intelligent design in Hume's Dialogues.

\section{The blueprint: Sellars' analysis of probability argu- ments}

The justification of IBdE in this paper is carried out in broad analogy to Sellars' analysis of inductive - or, as Sellars prefers to say: probability - arguments [cf. Sellars, 1969]. The purpose of this section is to make this alleged link explicit. The first thing to note is that every deductive argument $a=\left(P_{1}, \ldots, P_{n} ; C\right)$ with premisses $P_{1}, \ldots, P_{n}$ and conclusion $C$ can be turned into a higher order counterpart which infers that it is reasonable to accept $C$ given (i) the argument $a$ and (ii) the fact that its premisses are true. Consider for example the deductive argument below.

(B1) All electronic devices are man-made.

(B2) My phone is an electronic device.

(B3) Thus: My phone is man-made.

It corresponds to the following higher order counterpart.

(C1) (B1) and (B2) jointly imply (B3). (Argument B is valid.) 
(C2) (B1) and (B2) are true. (Its premisses are true.)

(C3) It is reasonable to accept a statement which is implied by a set of true statements.

(C4) Thus: It is reasonable to accept (B3).

Sellars claims, I understand, that when it comes to probabilistic arguments, the higher order counterpart is not merely a meta-reformulation of the more basic first order argument. On the contrary, it is the meta-argument which is fundamental and ultimately licenses inductive reasoning. The first order formulation of a probabilistic argument merely approximates the principal and underlying higher order argument. To illustrate this point, consider the following inductive argument.

(D1) Most professors have been abroad.

(D2) Peter is a professor.

(D3) Thus: (Probably,) Peter has been abroad.

Sellars' analysis amounts to saying that the second order counterpart to this argument actually reads,

(E1) Most of the statements of the form " $a$ has been abroad", where " $a$ " refers to some professor, are true. (D1)

(E2) "Peter" refers to some professor. (D2)

(E3) It is, all things considered, reasonable to accept a statement which belongs to a class of mostly true statements.

(E4) Thus: It is reasonable to accept (D3).

Interestingly, the higher order argument $\mathrm{E}$ does not refer to any (probabilistic) inferential relations that are made explicit by the first order argument D. (Note that (C1) does refer to inferential relations of argument $\mathrm{B}$.) That is the reason why Sellars can consistently suggest that the argument E is more basic than the argument $\mathrm{D}$ - and not the other way around. Actually, Sellars denies that there are genuine, independent probability arguments of the type D at all. Our inductive reasoning, he claims, has to be reconstructed as meta-reasoning.

Both meta-arguments $\mathrm{C}$ and D are practical arguments. They conclude that it is reasonable to do this or that - namely, to accept a certain statement. ${ }^{6}$ These actions are recommended with respect to a common epistemic aim. Sellars assumescompare premisses (C3) and (E3) - that it is reasonable to maximise the number of true beliefs as long as the error ratio can be controlled. In regard of this epistemic aim, it is reasonable to accept the conclusion of a probability argument. The

\footnotetext{
${ }^{6}$ This raises the question whether we can decide (what) to believe at all. Bernard Williams famously answered this question in the negative [Williams, 1973]. We cannot acquire beliefs at will, he convincingly argued, because beliefs aim at truth. Admittedly, I cannot decide to believe that $p$ while I am convinced that $\mathrm{p}$ is false. Our inability to decide to believe, however, does not undermine the practical meta-arguments we are studying presently. For these arguments try to demonstrate that adopting this or that belief is truth-conducive, or, more generally, furthers our epistemic aims. To consider such a practical meta-arguments as sound therefore doesn't assume that we're able to acquire arbitrary beliefs at will.
} 
normative strength of probability arguments stems solely from their higher order analysis.

Sellars' approach may serve as a blueprint for justifying IBdE. Without attempting to impose a deductive structure, the teleological argument can be reconstructed as,

(F1) All subdivisions of nature are adjusted to each other such that means are adapted to appropriate ends. (Explanandum $E$ )

(F2) The existence of God, an intelligent being that created the universe, is the best explanation for all subdivisions of nature being adjusted to each other such that means are adapted to appropriate ends. (Hypothesis $H^{*}$ explains $E$ best.)

(F3) Thus: God exists. (Explanans $H^{*}$ )

I suggest that this is, like the probability argument $\mathrm{D}$, not a genuine and independent reasoning, either. What stands behind this IBdE is the following practical metaargument.

(G1) Accepting that God exists maximises-compared to accepting any other alternative explanatory hypothesis-the robustness of one's position. (Accepting the best explanation $H^{*}$ maximises the robustness of one's position relative to all available explanatory hypotheses.)

(G2) It is reasonable to maximise the robustness of one's position.

(G3) One of the explanatory hypotheses should be accepted.

(G4) Thus: It is reasonable to accept that God exists.

Premiss (G1) states that accepting the best explanation is the comparatively best way to achieve a certain epistemic aim - maximising robustness. (G2) adds that it is reasonable to pursue that very aim while (G3) rules out the option of not accepting any explanatory hypothesis (i.e. of being agnostic). Argument $G$ is entirely independent of any inferential claim contained in F. Thus, the inference expressed by $\mathrm{F}$ (its normative strength) may be reduced to $\mathrm{G}$ in a non-circular way. Moreover, the reconstruction of IBdE as practical meta-syllogism helps to refine the original inference pattern of IBdE. Provided the reconstruction given by G can be consistently defended in the following sections, it demonstrates that any IBdE has to assume that (i) $H^{*}$ is, relative to a set of alternative explanations $H_{i}$, the best explanation of $E$, that (ii) $E$ is a fact, as well as that (iii) $E$ should be explained at all. For unless (iii) holds, it is not clear why premiss (G3) of the second order counterpart is true. And if the premisses of the corresponding practical meta-syllogism aren't true, the specific IBdE isn't warranted in the first place.

Hence, it remains to be shown that the meta-syllogism's premisses (G1) and (G2) are indeed true - given the premisses of the respective first order argument. The next section, by introducing the theory of dialectical structures, sets the stage for doing so. Subsequently, premiss (G2) will be spelled out and justified in section 4 . 


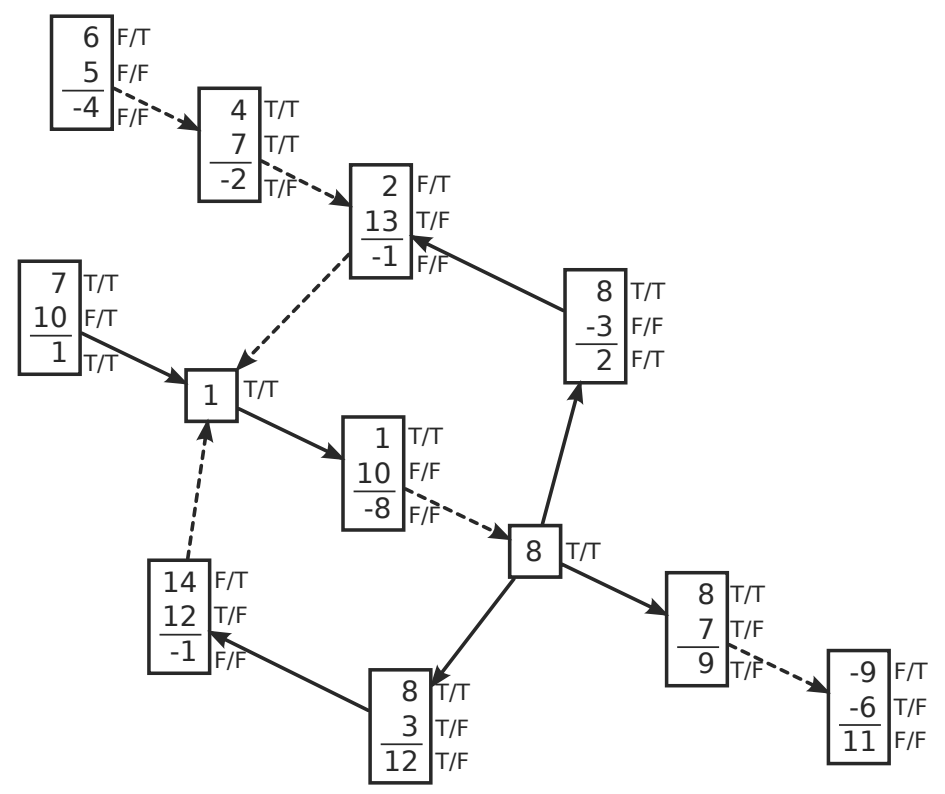

Figure 1: A dialectical structure with two complete positions attached. Truth values are symbolised by "T" (true) and "F" (false).

Thereupon, sections 5 and 6 explain why premiss (G1) is entailed by the premisses of the corresponding first order IBdE.

\section{The general framework: Theory of dialectical struc- tures}

A dialectical structure $\tau=\langle T, A, U\rangle$ is a set of deductive arguments (premissconclusion structure), $T$, on which an attack relation, $A$, and a support relation, $U$, are defined as follows $(a, b \in T)$ :

- $A(a, b): \Longleftrightarrow a$ 's conclusion is contradictory to one of $b$ 's premisses;

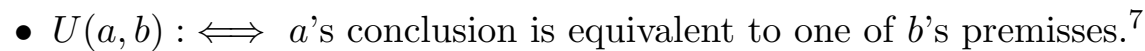

Complex debates can be reconstructed as dialectical structures. Likewise, a reconstruction of the controversial argumentation in Part 6 of Hume's Dialogues Concerning Natural Religion will be sketched in section 7. Figure 1 depicts a purely

\footnotetext{
${ }^{7} \mathrm{~A}$ dialectical structures is a special type of bipolar argumentation framework as developed by Cayrol and Lagasquie-Schiex [2005]. Cayrol and Lagasquie-Schiex extend the abstract approach of Dung [1995] by adding support-relations to Dung's framework which originally considered attackrelations between arguments only. A specific interpretation of Dung's abstract framework that analyses arguments as premiss-conclusion structures is carried out in Bondarenko et al. [1997]. The theory of dialectical structures is more thoroughly developed in Betz [2010a].
} 


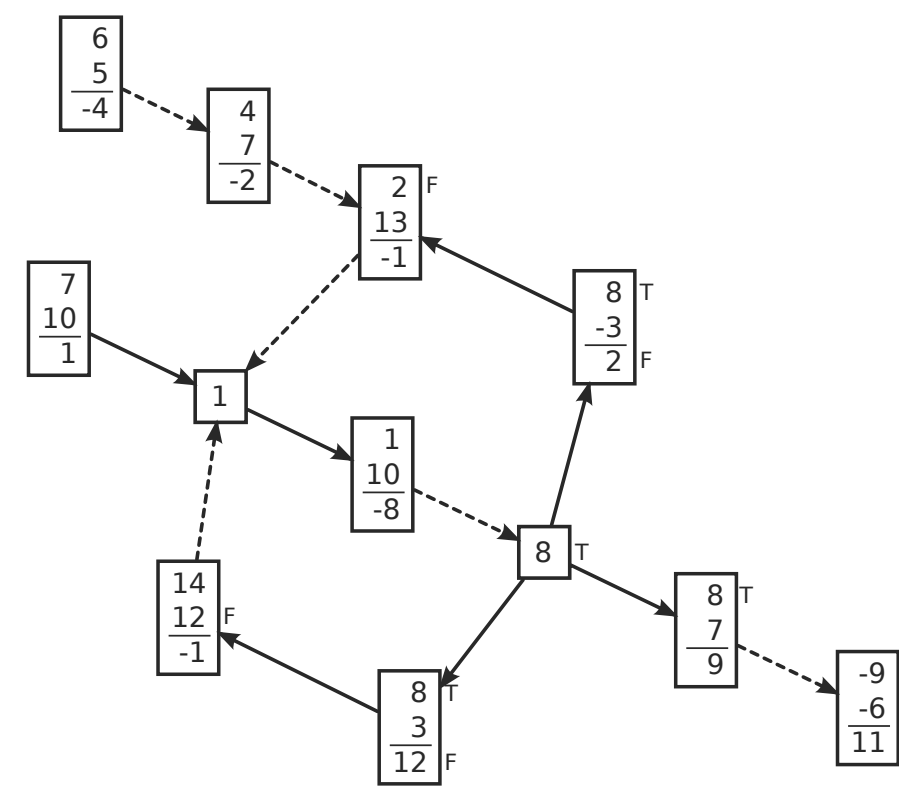

Figure 2: A dialectical structure with one partial positions attached.

formal example of a dialectical structure. Numbers stand for sentences, and a negative number denotes the negation of the sentence which is designated by the corresponding positive integer. Each box represents an argument or a thesis. Continuous and dashed arrows indicate the support and attack relationship, respectively.

Relative to a dialectical structure $\tau$, which in a sense depicts the state of a debate, one can specify the positions of different proponents. We may, generally, distinguish complete and partial positions. A complete position $\mathcal{Q}$ (a proponent can adopt) on $\tau$ is a truth value assignment to all sentences which figure in arguments in $T$, i.e. $\mathcal{Q}: S \rightarrow\{t, f\}$, where $S$ is the set of all sentences in $\tau$. A partial position $\mathcal{P}$ (a proponent can adopt) on $\tau$ is a truth value assignment to some sentences which figure in arguments in $T$, i.e. $\mathcal{P}: S^{\prime} \rightarrow\{t, f\}$, where $S^{\prime} \subseteq S$. Whereas figure 1 shows two complete positions defined on a dialectical structure, figure 2 gives an example for a partial position defined on the very same debate.

Partial positions can be combined. Let $\mathcal{P}_{1}: S_{1} \rightarrow\{t, f\}$ and $\mathcal{P}_{2}: S_{2} \rightarrow\{t, f\}$ be two partial positions which agree on $S_{1} \cap S_{2}$. The conjunction of these positions, $\left(\mathcal{P}_{1} \& \mathcal{P}_{2}\right): S_{1} \cup S_{2} \rightarrow\{t, f\}$, can be defined by,

$$
p \mapsto\left\{\begin{array}{ll}
\mathcal{P}_{1}(p) & \text { if } p \in S_{1} \\
\mathcal{P}_{2}(p) & \text { if } p \in S_{2} \backslash S_{1}
\end{array} .\right.
$$

Obviously, the arguments that make up a dialectical structure impose certain constraints on what a proponent can reasonably assert. Not every complete or partial position can rationally be adopted. Thus, a complete position $\mathcal{Q}$ on $\tau$ is 
(dialectically) coherent if and only if

1. equivalent sentences are assigned the same truth value;

2. contradictory sentences are assigned complementary truth values;

3. if every premiss $p$ of some argument $a \in T$ is assigned the value "true", then $a$ 's conclusion is assigned the value "true", too.

A partial position $\mathcal{P}: S^{\prime} \rightarrow\{t, f\}$ on $\tau$ is (dialectically) coherent if and only if it can be extended to a complete position $\mathcal{Q}$ on $\tau\left(\mathcal{P}=\left.\mathcal{Q}\right|_{S^{\prime}}\right)$ which is coherent.

Returning to the complete positions depicted in figure 1, we may note:

- The left-hand-side complete position in that example is coherent. It complies with the coherence conditions set up above.

- The right-hand-side complete position is, however, not coherent. Yet, that position does not merely defy one coherence constraint. Actually, all three conditions are violated. Thus, the right-hand-side position violates constraint (1) because the tokens of sentence 10 are assigned different truth values; it violates constraint (2) because the contradictory sentences 3/-3 are both considered true; and it violates constraint (3) because the conclusion of argument $(4,7 ;-2)$ is false although its premisses are true.

Moreover, the partial position shown in figure 2 is not coherent, either, since it cannot be extended to a complete, coherent position. To see this, consider, first of all, the argument $(8,-3 ; 2)$. Since premiss 8 is true and the conclusion 2 is false, the remaining premiss -3 has to be false. Otherwise the complete position would violate the third constraint. Secondly, in regard of argument $(8,3 ; 12)$, sentence 3 must be false for analogous reasons. Yet sentences 3 and -3 cannot be false in the same time because of the second constraint. Hence, the partial position cannot be extended to a coherent complete position.

\section{The epistemic aim of robustness}

It is a central claim of our analysis that accepting the best explanation as true is reasonable with respect to the epistemic aim of maximising the robustness of one's point of view. We are now in a position to introduce this specific epistemic aim explicitly. ${ }^{8}$ Generally, proponents can, while engaging in controversial argumentation, pursue different discursive aims such as, e.g., ensuring that one's position is (dialectically)

\footnotetext{
${ }^{8}$ See, for a much more detailed discussion of the concept of robustness along the lines of this section, Betz [2011].
} 
coherent, or demonstrating that the position of an opponent is not. ${ }^{9}$ To adopt a robust partial position is a rational discursive aim, too. The argumentation-theoretic notion of robustness signifies, informally, that the respective partial position can be coherently adopted independently of how truth values are assigned to the sentences outside that partial position. A robust partial position $\mathcal{P}$

- can be extended in very different ways to a complete, coherent position,

- hardly determines the truth values of sentences outside $\mathcal{P}$,

- is therefore immune to falsification by fixing truth values outside $\mathcal{P}$.

On the opposite, a position $\mathcal{P}$ which is not robust

- can be extended in very few ways to a complete, coherent position,

- more or less fully determines the truth values of sentences outside $\mathcal{P}$,

- is consequently prone to falsification by fixing truth values outside $\mathcal{P}$.

A robust partial position, thus understood, is thence comparatively resilient against future criticism and is likely to remain coherent throughout a controversial debate. Note that this argumentation-theoretic concept should be distinguished from the methodological notion of robustness, which pertains, e.g., to scientific models or predictions. A model is typically said to be robust if its results don't depend sensitively on initial and boundary conditions, or if it has been successfully applied in very different kinds of contexts. ${ }^{10}$

This said, it is - given the debate is controversial, and ongoing - a reasonable discursive aim to possess a rather robust partial position. Striving for a robust position is simply motivated by avoiding incoherency. ${ }^{11}$ It is quite clear, from what has been said so far, that robustness is a comparative concept. A partial position, $\mathcal{P}$, can be more (or less) robust than another one. A quantitative indicator of a partial position's robustness in line with the informal explication of that notion above is given by the ratio,

number of coherent \& complete positions on $\tau$ that extend $\mathcal{P}$ number of coherent \& complete positions on $\tau$

\footnotetext{
${ }^{9}$ On the variety of discursive aims and their role in evaluating argumentative actions see also Betz [2010b].

${ }^{10}$ It would be intriguing to pursue the question in how far the methodological concept of robustness might be reduced to the argumentation-theoretic one. Here, the idea of invariance to auxiliary assumptions might provide a common denominator. Still, unfolding such an analysis goes beyond the scope of this paper.

${ }^{11} \mathrm{~A}$ formal investigation of the concept of robustness in Betz [forthcoming], based on multi-agent simulations of debate dynamics, suggests that robustness is also an indicator of truthlikeness. These results, if actually correct, could substantially strengthen the case for this paper's account of IBdE.
} 
The concept of a position's robustness is closely related to the notion of degree of partial entailment. Following Wittgenstein's basic idea in the Tractatus (and identifying cases with complete and coherent positions on $\tau$ ), the degree of partial entailment of a partial position $\mathcal{P}_{1}$ by a partial position $\mathcal{P}_{2}$, can be defined as,

$$
\begin{aligned}
\operatorname{Doj}\left(\mathcal{P}_{1} \mid \mathcal{P}_{2}\right)= & \frac{\text { number of cases with } \mathcal{P}_{1} \& \mathcal{P}_{2}}{\text { number of cases with } \mathcal{P}_{2}} \\
\text { number of complete } \& \text { coherent positions that } & \text { extend } \mathcal{P}_{1} \& \mathcal{P}_{2} \\
\text { number of complete } \& \text { coherent positions that } & \text { extend } \mathcal{P}_{2}
\end{aligned} .
$$

Degrees of partial entailment satisfy, under certain conditions which we shall assume to hold ${ }^{12}$, the axioms of probability theory.

Finally, the degree of justification of a partial position $\mathcal{P}$ can be defined as its degree of partial entailment from the empty set,

$$
\begin{aligned}
\operatorname{Doj}(\mathcal{P}) & =\operatorname{Doj}(\mathcal{P} \mid \emptyset) \\
& =\frac{\text { that extend } \mathcal{P}}{\text { number of complete } \& \text { coherent positions }} \\
& =\text { indicator of } \mathcal{P} \text { 's robustness. }
\end{aligned}
$$

\section{Reconstructing explanations as arguments in dialec- tical structures}

Explanations can be reconstructed as arguments. The explanandum, E, figures as conclusion of the thus reconstructed explanation, the explanans, $H$, as one premiss among others. This is, at least insofar as we disregard statistical explanations, a safe assumption. It is not to be confused with the stronger claim that every argument embodies an explanation (for its conclusion). Consider for example the explanation of nature's sophisticated internal adjustment by intelligent design (cf. premiss A2). This explanation might be reconstructed as follows.

(H1) God, i.e. an intelligent being that created the universe, exists. (Central explanatory hypothesis)

\footnotetext{
${ }^{12}$ This is the problem: For every probability measure over a set of statements, it holds that $P(A \vee B)=P(A)+P(B)$ for contrary $A, B$. Now assume that the three sentences $A \vee B, A$ and $B$ figure in some $\tau$ and that there is no dialectically coherent position according to which both $A$ and $B$ are true. Still, this does not guarantee that the (unconditional) degrees of partial entailment of $A$ and $B$ add up to the (unconditional) degree of partial entailment of $A \vee B$. That is because not every coherent complete position according to which $A$ is true assigns $A \vee B$ the value "true" - unless an argument like $(A ; A \vee B)$ is included in $\tau$. Thus, degrees of partial entailment satisfy the probability axioms only if the respective dialectical structure is suitably augmented by simple arguments as indicated.
} 
(H2) God is perfect.

(H3) Perfect beings create but perfect objects.

$(\mathrm{H} 4)$ Order is a perfection, i.e. being internally adjusted such that means are adapted to appropriate ends contributes to an object being closer to perfection.

(H5) Thus: All subdivisions of nature are adjusted to each other such that means are adapted to appropriate ends. (Explanans)

Explanatory hypotheses typically require additional assumptions in order to entail the explanans. In our example, the thesis of God's existence needs to be supplemented by premisses (H2)-(H4). Depending on the more general context of a debate, such auxiliary assumptions can be highly controversial, or, on the opposite, belong to the agreed-upon background knowledge. I suggest - and back up in the following section - that the status of these auxiliary assumptions determines, as one factor amongst others, the overall quality of the respective explanation. All other things being equal, the explanatory hypothesis which requires less (disputable) auxiliary assumptions in order to entail the explanans yields the better explanation.

In addition to the example of intelligent design above, we shall, in the remainder of this section, consider two abstract, yet more complex illustrations. Assume, firstly, that three individual facts, $E_{1} \ldots E_{3}$, were to be explained. Two rival hypotheses, $H_{1}$ and $H_{2}$, have been proposed to render these facts intelligible. $H_{1}$ and $H_{2}$ are incompatible, that is $H_{1}$ implies, given the background knowledge, $\neg H_{2}$. Apart from that, both hypotheses are on a par: Each one is able to explain the facts by making use of one auxiliary assumption which is not part of the background knowledge, and each one is itself backed by an argument which rests but on a single additional assumption. The resulting dialectical structure for this first, symmetrical example is displayed in figure 3 .

Given the auxiliary assumptions, the explanatory hypotheses strictly entail the evidence. Yet, some of these auxiliary premisses are, by construction of this case, not part of the background knowledge. Leaving the truth value of these additional assumptions open, we can nevertheless calculate the degree of partial entailment of the evidence by the alternative hypotheses. In order to do so, we define partial positions as follows.

$$
\begin{aligned}
\mathcal{H}_{1} & :=H_{1} \text { is true, } H_{2} \text { is false, } \\
\mathcal{H}_{2} & :=H_{1} \text { is false, } H_{2} \text { is true, } \\
\mathcal{H}_{0} & :=H_{1} \text { and } H_{2} \text { are false, } \\
\mathcal{E} & :=E_{1}, E_{2} \text { and } E_{3} \text { are true. }
\end{aligned}
$$




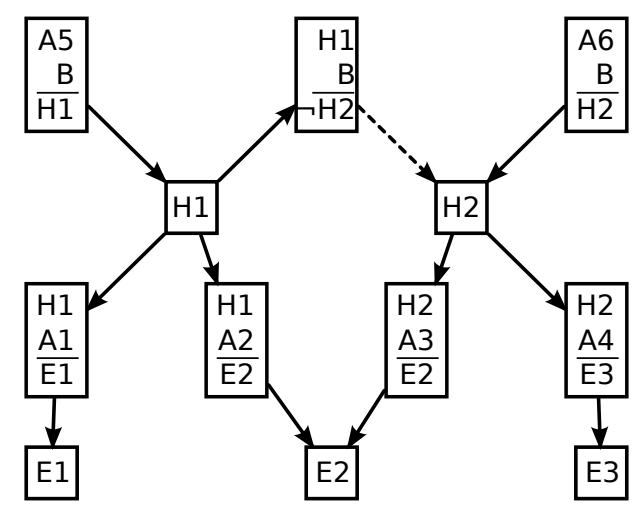

Figure 3: Dialectical structure depicting a symmetrical situation. The two explanatory hypotheses $H_{1}$ and $H_{2}$ are both capable of explaining the evidence $E_{1}, \ldots, E_{3}$. " $B$ " indicates statements which belong to the background knowledge and are, accordingly, assumed to be true. The $A_{i}$ s denote auxiliary assumption which don't belong to the background knowledge.

This allows us to calculate the following degrees of partial entailment. ${ }^{13,14}$

$$
\begin{aligned}
& \operatorname{DoJ}\left(\mathcal{E} \mid \mathcal{H}_{1}\right)=0.22 \\
& \operatorname{DoJ}\left(\mathcal{E} \mid \mathcal{H}_{2}\right)=0.22 \\
& \operatorname{DoJ}\left(\mathcal{E} \mid \mathcal{H}_{0}\right)=0.16
\end{aligned}
$$

As expected, because of the symmetry, the two hypotheses entail the explanans to the same degree. $\mathcal{H}_{0}$, which takes both hypotheses to be false, implies the evidence $\mathcal{E}$ to a significantly lower degree.

Consider a further example. Now, two individual facts, $E_{1}$ and $E_{2}$, are to be explained. Again, two rival, incompatible hypotheses have been proposed to explain the evidence. While both hypotheses have a similar backing, they fare quite differently in terms of explaining $E_{1}$ and $E_{2}$. While $H_{1}$ offers two simple explanations for the evidence, $H_{2}$ generates a rather tedious explanation for $E_{2}$, and no explanation for $E_{1}$ at all. All this translates into two simple arguments for $E_{1}$ and $E_{2}$, both taking off at $H_{1}$ and relying, on top of that, but on background assumptions. Moreover, we have a relatively long chain of reasoning that starts from $H_{2}$ and infers $E_{2}$ by using a couple of further auxiliary assumptions. Figure 4 shows the corresponding dialectical structure.

\footnotetext{
${ }^{13}$ It is tacitly understood that all degrees of partial entailment are, in the following, calculated relative to the background knowledge $B$. So, more precisely, $\operatorname{DoJ}(E \mid H)$ refers to $\operatorname{DoJ}(E \mid H \& B)$, and $\operatorname{Dou}(H)$ reads, fully spelled out, $\operatorname{DoJ}(H \mid B)$, for example.

${ }^{14}$ For the numerical calculation of these as well as all degrees of justification henceforth, compare the supplementary material.
} 


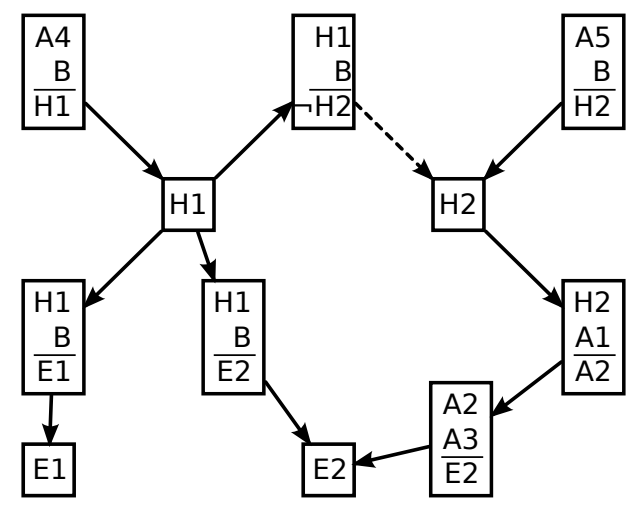

Figure 4: Dialectical structure depicting an asymmetrical situation. Hypothesis $H_{1}$ provides two neat explanations for the evidence. Its rival $H_{2}$, however, merely explains $E_{2}$, and this in a rather complicated or disputable way.

Once more, the rival explanatory hypotheses entail the evidence to a certain degree. We define:

$$
\begin{aligned}
\mathcal{H}_{1} & :=H_{1} \text { is true, } H_{2} \text { is false } \\
\mathcal{H}_{2} & :=H_{1} \text { is false, } H_{2} \text { is true } \\
\mathcal{H}_{0} & :=H_{1} \text { and } H_{2} \text { are false } \\
\mathcal{E} & :=E_{1} \text { and } E_{2} \text { are true. }
\end{aligned}
$$

This allows us to calculate the following degrees of partial entailment.

$$
\begin{aligned}
\operatorname{DoJ}\left(\mathcal{E} \mid \mathcal{H}_{1}\right) & =1 \\
\operatorname{DoJ}\left(\mathcal{E} \mid \mathcal{H}_{2}\right) & =0.3 \\
\operatorname{DoJ}\left(\mathcal{E} \mid \mathcal{H}_{0}\right) & =0.29
\end{aligned}
$$

Hence, the hypothesis $H_{1}$ strictly implies the explanans (obviously so, as the reconstructed explanations merely rely on background assumption that are assumed to be true). Its rival $H_{2}$, however, implies the evidence $\mathcal{E}$ to a much lower degree. Actually, the degree of partial entailment of the explanans by $H_{2}$ is hardly higher than by the position according to which neither hypothesis is true.

The graph-theoretical analysis of rival explanations set forth in this section exhibits a structural analogy to Paul Thagard's theory of explanatory coherence [Thagard, 1992, chapter 4]. This is because Thagard, too, represents concrete explanatory situations as graphs which lay out the explanatory relations between different hypotheses and items of evidence. In contrast to Thagard's theory, however, the approach presented in this paper doesn't take the explanatory relations as primi- 
tive, but reduces them to inferential relations between the respective statements so that logico-dialectical considerations appertain to the analysis. As we will see in the following section, this allows us to justify IBdE on argumentation-theoretic grounds.

\section{Accepting the best explanation maximises robustness}

This section establishes premiss (G1) of the practical meta-syllogism which underlies every IBdE, i.e. it demonstrates that accepting the best explanation - relative to adopting any rival explanatory hypothesis - maximises one's partial position's robustness. The core of the argument can be put as follows: Assume we were to adopt one of the alternative explanatory partial positions $\mathcal{H}_{1}, \ldots, \mathcal{H}_{n}$ which attempt to explain the evidence $\mathcal{E}$, thus extending our prior partial position $\mathcal{Q}$. Let us denote the background knowledge by $\mathcal{B}$. Provided that the epistemic aim which guides our choice is to maximise the robustness of our new position, we have to adopt $\mathcal{H}_{k}$ $(1 \leq k \leq n)$ such that

$$
\operatorname{DoJ}\left(\mathcal{H}_{k} \& \mathcal{Q} \mid \mathcal{E} \& \mathcal{B}\right)=\max _{i=1 \ldots n} \operatorname{DoJ}\left(\mathcal{H}_{i} \& \mathcal{Q} \mid \mathcal{E} \& \mathcal{B}\right)
$$

But since we have, with Bayes' theorem,

$$
\begin{aligned}
\operatorname{DoJ}\left(\mathcal{H}_{i} \& \mathcal{Q} \mid \mathcal{E} \& \mathcal{B}\right) & =\operatorname{Dov}\left(\mathcal{H}_{i} \mid \mathcal{E} \& \mathcal{B} \& \mathcal{Q}\right) \times \underbrace{\operatorname{DoJ}(\mathcal{Q} \mid \mathcal{E} \& \mathcal{B})}_{\text {const. }} \\
& \propto \frac{\operatorname{DoJ}\left(\mathcal{E} \mid \mathcal{H}_{i} \& \mathcal{B} \& \mathcal{Q}\right) \times \operatorname{DoJ}\left(\mathcal{H}_{i} \mid \mathcal{B} \& \mathcal{Q}\right)}{\underbrace{\operatorname{DoJ}(\mathcal{E} \mid \mathcal{B} \& \mathcal{Q})}_{\text {const. }}} \\
& \propto \operatorname{DoJ}\left(\mathcal{E} \mid \mathcal{H}_{i} \& \mathcal{B} \& \mathcal{Q}\right) \times \operatorname{DoJ}\left(\mathcal{H}_{i} \mid \mathcal{B} \& \mathcal{Q}\right),
\end{aligned}
$$

it remains but to be shown that the product in equation 9 is maximal for the best explanation.

The argument in the remainder of this section proceeds, generally spoken, in four steps: First, we spell out the comparative concept of a better ("lovelier") explanation. Secondly, we show that whatever contributes to some hypothesis $H_{1}$ being a better explanation than $H_{2}$ is reflected in certain features of the dialectical structure that reconstructs the respective explanations. Thirdly, we argue that these features increase either the likelihood of the evidence given the hypothesis, $\operatorname{DoJ}\left(\mathcal{E} \mid \mathcal{H}_{i} \& \mathcal{B} \& \mathcal{Q}\right)$, or the prior degree of justification of the respective hypothesis, $\operatorname{DoJ}\left(\mathcal{H}_{i} \mid \mathcal{B} \& \mathcal{Q}\right)$. So we can, finally, conclude that the best explanation maximises the enlarged position's robustness, indeed.

This general reasoning has to be carried out in a piecemeal fashion. Thus we identify, in the following, different explanatory qualities which characterise a good explanation and demonstrate for each of these how they increase either the likeli- 
hood of the evidence given the hypothesis or the prior degree of justification of the respective hypothesis.

Scope. If $H_{1}$ explains more pieces of evidence than $H_{2}$, then $H_{1}$ is ceteris paribus a better explanation than $\mathrm{H}_{2}$. What does this mean in terms of the reconstructed dialectical structure? Assume that $H_{1}$ explains $E_{1}, \ldots, E_{n}$ whereas $H_{2}$ explains but $E_{1}, \ldots, E_{m}$ with $n>m$. Let each individual explanation rely on one disputable auxiliary assumption. So we have $n$ arguments starting from $H_{1}$ and $m$ arguments with premiss $H_{2}$. But then, as is intuitively clear, the total evidence $\mathcal{E}$ (including $E_{1}, \ldots, E_{n}$ ) is entailed by $H_{1}$ to a higher degree than by $H_{2} \cdot{ }^{15}$ Hence $\operatorname{DoJ}\left(\mathcal{E} \mid \mathcal{H}_{1} \& \mathcal{B} \& \mathcal{Q}\right)>\operatorname{DoJ}\left(\mathcal{E} \mid \mathcal{H}_{2} \& \mathcal{B} \& \mathcal{Q}\right)$.

Precision. If $H_{1}$ explains the same phenomena as $H_{2}$, yet with a much higher degree of precision, then $H_{1}$ is ceteris paribus a better explanation than $H_{2}$. This situation may be reconstructed as follows. Let $E_{1}$ and $E_{2}$ state two facts about one and the same phenomenon. $H_{1}$ explains $E_{1} ; H_{2}$ explains $E_{2}$. But assume that $E_{1}$ is a much more detailed and specific statement than $E_{2}$. As a consequence, $E_{1}$ entails (given appropriate background assumptions) $E_{2}$. Therefore, $H_{1}$ partially entails (and explains) both pieces of evidence whereas $H_{2}$ merely explains one. $H_{1}$ is thus superior in terms of scope and, according to the previous point, we thence have $\operatorname{DoJ}\left(\mathcal{E} \mid \mathcal{H}_{1} \& \mathcal{B} \& \mathcal{Q}\right)>\operatorname{Dov}\left(\mathcal{E} \mid \mathcal{H}_{2} \& \mathcal{B} \& \mathcal{Q}\right)$.

Simplicity. If the explanations based on $H_{1}$ are simpler than those based on $H_{2}$, $H_{1}$ is ceteris paribus a better explanation than $H_{2}$. An explanation is simple, if it relies on few auxiliary assumptions; it is, on the contrary, complicated, if many disputable assumptions are involved. ${ }^{16}$ This characteristic, it seems to me, is straightforwardly translated into the number of auxiliary assumptions the reconstruction of an explanation has to make use of. Thus, the simpler an explanation, the fewer disputable premisses figure in its reconstruction (cf. the example given in figure 4.). Consequently, an explanatory hypothesis which gives rise to a simpler explanation entails the explanandum, ceteris paribus, to a higher degree. Therefore, $\operatorname{DoJ}\left(\mathcal{E} \mid \mathcal{H}_{1} \& \mathcal{B} \& \mathcal{Q}\right)>\operatorname{DoJ}\left(\mathcal{E} \mid \mathcal{H}_{2} \& \mathcal{B} \& \mathcal{Q}\right)$.

Unificatory strength. If $H_{2}$ explains closely related and homogeneous pieces of evidence, whereas $H_{1}$ can explain previously unrelated and heterogeneous evidence, $H_{1}$ is ceteris paribus a better explanation than $H_{2}$. To understand

\footnotetext{
${ }^{15}$ To make this more explicit, note that the number of complete and coherent positions that extend $\mathcal{E} \& \mathcal{H}_{1}$ equals the number of those positions that extend $\mathcal{E} \& \mathcal{H}_{2}$. So the likelihood merely depends on the number of positions which extend the respective hypotheses (see equation 4). But because the reconstructed explanations impose $n$ constraints on $\mathcal{H}_{1}$ but only $m<n$ constraints on $\mathcal{H}_{2}$, strictly more positions extend $\mathcal{H}_{2}$ than $\mathcal{H}_{1}$. Thus $\operatorname{DoJ}\left(\mathcal{E} \mid \mathcal{H}_{1}\right)>\operatorname{DoJ}\left(\mathcal{E} \mid \mathcal{H}_{2}\right)$.

${ }^{16}$ Note that the simplicity of an explanation should be distinguished from the simplicity of the explanatory hypothesis on which the explanation is based.
} 


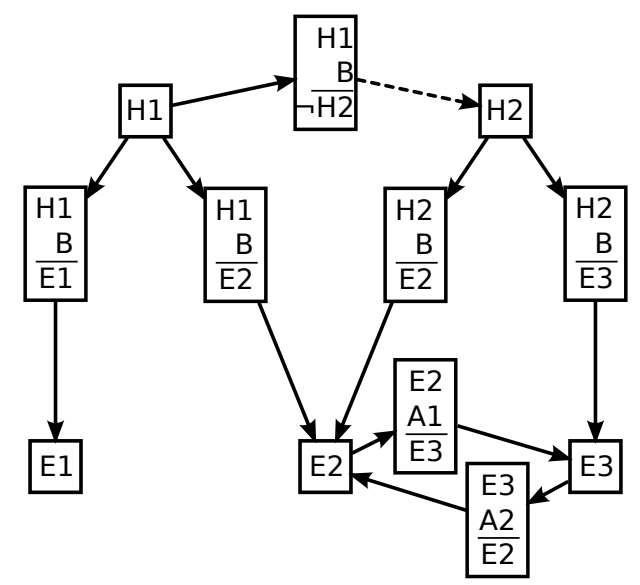

Figure 5: Dialectical structure depicting a paradigmatic example for the impact of unificatory strength. Hypothesis $\mathrm{H}_{2}$ provides two neat explanations for homogeneous evidence. Its rival $H_{1}$, however, explains unrelated evidence $E_{1}$ and $E_{2}$, thereby unifying heterogeneous facts.

the role of unificatory strength, consider the following case. Assume that $H_{1}$ and $H_{2}$ explain the 'same amount of' evidence, say $H_{1}$ explains $E_{1}$ and $E_{2}$ whereas $H_{2}$ explains $E_{2}$ and $E_{3}$. On top of that, assume $E_{2}$ and $E_{3}$ represent homogeneous evidence while $E_{1}$ is entirely unrelated to $E_{2}$ (and $E_{3}$ ). In terms of the dialectical structure, this means that $E_{2}$ and $E_{3}$ are related by certain arguments, whereas $E_{1}$ bears in no such inferential relations. Figure 5 depicts the entire dialectical situation. In this paradigmatic case, the evidence $\mathcal{E}$ is entailed by $H_{1}$ to a significantly higher degree (0.67) than by $H_{2}(0.5)$. The general reason for this is that $H_{1}$ indirectly entails $E_{3}$, too, while $H_{2}$, explaining but homogeneous evidence, does not entail the unrelated $E_{1}$. In sum, we thus see why unificatory strength increases the likelihood of the explanandum, $\operatorname{DoJ}\left(\mathcal{E} \mid \mathcal{H}_{1} \& \mathcal{B} \& \mathcal{Q}\right)>\operatorname{DoJ}\left(\mathcal{E} \mid \mathcal{H}_{2} \& \mathcal{B} \& \mathcal{Q}\right)$.

Prima facie plausibility. If the explanatory hypothesis $H_{1}$ has, prima facie, a higher plausibility than $H_{2}, H_{1}$ is ceteris paribus a better explanation than $H_{2}$. Yet, if $H_{1}$ is prima facie more plausible than $H_{2}$, this signals that there are, independently of the evidence $\mathcal{E}$, more and (relative to the background knowledge, $\mathcal{B}$, and one's previous convictions, $\mathcal{Q}$ ) better arguments in favour of $H_{1}$ than in favour of $H_{2}$. Or, the other way around, $H_{2}$ faces more severe criticism than $H_{1}$. In either way, these independent arguments directly lead to the degree of justification of $H_{1}$ being greater than that of $H_{2}$ [cf. Betz, 2011]. Thence, $\operatorname{Dou}\left(\mathcal{H}_{1} \mid \mathcal{B} \& \mathcal{Q}\right)>\operatorname{DoJ}\left(\mathcal{H}_{2} \mid \mathcal{B} \& \mathcal{Q}\right)$.

Let us sum up! We have found that the properties which make an explanatory hypothesis a lovely one increase either the degree of partial entailment of the 


\begin{tabular}{|c|c|c|c|c|c|}
\hline & $\operatorname{DoJ}\left(\mathcal{E} \mid \mathcal{H}_{i}\right)$ & $x$ & $\operatorname{Dos}\left(\mathcal{H}_{i}\right)$ & $\propto$ & $\operatorname{DoJ}\left(\mathcal{H}_{i} \mid \mathcal{E}\right)$ \\
\hline$i=1$ & 0.22 & & 0.35 & & 0.4 \\
\hline$i=2$ & 0.22 & & 0.35 & & 0.4 \\
\hline$i=0$ & 0.16 & & 0.31 & & 0.2 \\
\hline
\end{tabular}

Table 1: Degrees of partial entailment for the dialectical structure shown in figure 3.

\begin{tabular}{|c|c|c|c|c|c|}
\hline & $\operatorname{DoJ}\left(\mathcal{E} \mid \mathcal{H}_{i}\right)$ & $x$ & $\operatorname{DoJ}\left(\mathcal{H}_{i}\right)$ & $\propto$ & $\operatorname{DoJ}\left(\mathcal{H}_{i} \mid \mathcal{E}\right)$ \\
\hline$i=1$ & 1 & & 0.19 & & 0.44 \\
\hline$i=2$ & 0.3 & & 0.48 & & 0.33 \\
\hline$i=0$ & 0.29 & & 0.33 & & 0.22 \\
\hline
\end{tabular}

Table 2: Degrees of partial entailment for the dialectical structure shown in figure 4 .

explanandum by the hypothesis, $\operatorname{DoJ}\left(\mathcal{E} \mid \mathcal{H}_{i} \& \mathcal{B} \& \mathcal{Q}\right)$, or the degree of justification of the hypothesis itself, $\operatorname{DoJ}\left(\mathcal{H}_{i} \mid \mathcal{B} \& \mathcal{Q}\right)$. This seems to provide sufficient reason to stipulate that some $H_{1}$ constitutes a strictly better explanation than $H_{2}$ iff $H_{1}$ maximises the product of those two terms. Note that such an explication of the notion of the best explanation allows for weighing the different explanatory qualities against each other: E.g., if some hypothesis $H_{1}$ substantially surpasses $H_{2}$ with respect to scope, precision, and unificatory strength while being only slightly inferior in terms of prima facie plausibility, $H_{1}$ obviously counts as the lovelier explanation-and this corresponds to the fact that the product of $\operatorname{DoJ}\left(\mathcal{E} \mid \mathcal{H}_{i} \& \mathcal{B} \& \mathcal{Q}\right)$ and $\operatorname{DoJ}\left(\mathcal{H}_{i} \mid \mathcal{B} \& \mathcal{Q}\right)$ is greater for $H_{1}$ than for $H_{2}$. As an immediate consequence, the best explanationcall it $H^{*}$ - of a set of alternative hypotheses thus satisfies equation 8 . As we have seen, this means that adopting the best explanation maximises the robustness of one's (extended) position. Thence, we have justified the general premiss (G1) of the practical meta-argument which underlies every inference to the best (deductive) explanation.

Let us apply these findings to the formal examples we have studied in the previous section. Consider the case of the two symmetric explanations depicted in figure 3. Table 1 details the degrees of partial entailment of the respective partial positions. As the last column shows, extending one's initial partial position by $\mathcal{H}_{1}$ yields as robust a new position as accepting $\mathcal{H}_{2}$. In regard of the symmetry between the explanatory hypothesis, this is not surprising. We see, moreover, that enlarging one's position by assigning both hypotheses the truth-value "false", $\mathcal{H}_{3}$, results in a significantly less robust position. With a view to maximising robustness, one of the two equally lovely explanations should be accepted. Let us turn to the second, asymmetric example displayed in figure 4 . Table 2 reports the corresponding degrees of partial entailment. We had previously assumed that in this example, $H_{1}$ clearly provides the better explanation. This is reflected in the degree of partial entailment of the evidence. Still, in terms of unconditional degree of justification, $H_{1}$ appears to 
be worse off than $H_{2}$ (0.19 vs. 0.48). ${ }^{17}$ This relative shortcoming of $H_{1}$ is, however, outweighed by its success in entailing the explanandum, as the last column shows. Accepting $H_{1}$ (instead of $H_{2}$ ) as true, maximises the robustness of the accordingly extended position. Moreover, considering both hypotheses as false would be the worst thing to do with regard to fostering robustness. Inference to the best deductive explanation, i.e. to $H_{1}$, is justified in this second example, too.

\section{Applying the account}

\subsection{Semmelweis's explanation of childbed fever}

Ignaz Semmelweis's investigation into the causes of childbed fever in the mid 19th century has been prominently used as a case study in philosophy of science [Hempel, 1966, pp. 3-8]. Here, I follow closely Lipton's analysis of this example [Lipton, 2004, pp. 74ff.] in order to show that Semmelweis's abductive inferences can be justified in line with the dialectic account of IBdE set forth in this paper.

Comparing two maternity divisions in his Viennese hospital, Semmelweis observed that the proportion of women who contracted childbed fever varied substantially between the two groups. This represented the main observation Semmelweis tried to understand.

$\left(E_{1}\right)$ More women contract childbed fever in the first than in the second division.

Semmelweis considered three types of hypotheses that presumably identify causes of childbed fever. The hypotheses of the first type suggest causes that actually didn't vary between the two divisions, such as, e.g., epidemic influences, which would effect entire city districts in similar ways,

(EP) The outbreak of childbed fever is controlled by epidemic influences.

This hypothesis, however, neither explained the observed differences $\left(E_{1}\right)$, nor were its particular observational implications, such as variations between different hospitals (we abbreviate: $E_{5}, E_{6}$ ), verified by Semmelweis.

In contrast, the hypotheses of the second type do refer to certain differences between the two divisions. But Semmelweis judged that the specific differences

\footnotetext{
${ }^{17}$ This is, to a certain degree, an artificial effect due to the tightly restricted example. The arguments which primarily determine, according to our example, the degrees of justification are the reconstructed explanations themselves. Yet, every observational implication of a hypothesis represents eo ipso a potential counter-argument and thus reduces, according to the dialectical analysis, its unconditional degree of justification. In a dialectical structure which consists mainly of the reconstructed explanations, the explanatory hypothesis with the greater scope, precision, and unificatory strengths will therefore necessarily possess the lower prima facie degree of justification. This effect would, however, vanish if a sufficiently large part of the entire debate were reconstructed such that arguments which are independent of the explanandum $\mathcal{E}$ dominated the unconditional degrees of justification.
} 
these hypotheses stress were in fact negligible. A major difference between the two groups was, for instance, that medical students carried out some examinations in the first division whereas midwives were trained in the second division. The rough examination hypothesis claimed,

(RE) The outbreak of childbed fever is controlled by injuries that stem from rough examinations.

While this hypothesis may yield an explanation for $E_{1}$, Semmelweis thought that the particular assumptions the explanation rests on $\left(A_{1}\right)$ are rather weak: for one thing, midwives are equally imprecise or rough as medical students $\left(A_{5}\right)$; secondly, the injuries caused by childbirth itself are significantly greater than those due to examinations $\left(A_{6}\right)$. In addition, further empirical implications of the rough examination hypothesis $\left(E_{4}\right)$ were not confirmed.

The hypothesis that childbed fever was caused by the (accidental) injection of cadavric substance into the blood stream made up a third category of potential explanations.

(CH) The outbreak of childbed fever is controlled by the degree of contamination with cadavric substance.

Since medical students typically came in contact with cadavric substance, whereas midwives didn't, $\mathrm{CH}$ provided an explanation for the observed difference between the divisions $\left(E_{1}\right)$. Moreover, $\mathrm{CH}$ explained why the difference vanishes once the students disinfect their hands before examining the women in the first division $\left(E_{2}\right)-\mathrm{a}$ fact verified after Semmelweis gave according instructions. Finally, CH could also explain why a colleague of Semmelweis suffered from symptoms similar to those of childbed fever, and eventually died, after being slightly wounded at his finger during an autopsy $\left(E_{3}\right)$.

Figure 6 provides the dialectical structure which contains the reconstructed explanations, arguments, theoretical hypotheses and pieces of evidence relevant to Semmelweis's investigation. ${ }^{18}$ The hypotheses explain (and imply) different observational statements. Only $E_{1}, E_{2}$ and $E_{3}$, however, were actually observed. $\mathrm{CH}$ explains this diverse evidence fully and provides the comparatively best explanation. The explanation of $E_{1}$ by $R E$ suffers from implausible assumptions, which is made explicit through counter-arguments against $A_{1}$. We consider the following partial positions.

$$
\begin{aligned}
& \mathcal{H}_{1}:=(\mathrm{EP}) \text { is true, } \\
& \mathcal{H}_{2}:=(\mathrm{RE}) \text { is true, }
\end{aligned}
$$

\footnotetext{
${ }^{18}$ We assume implicitly that the three hypotheses are mutually exclusive.
} 


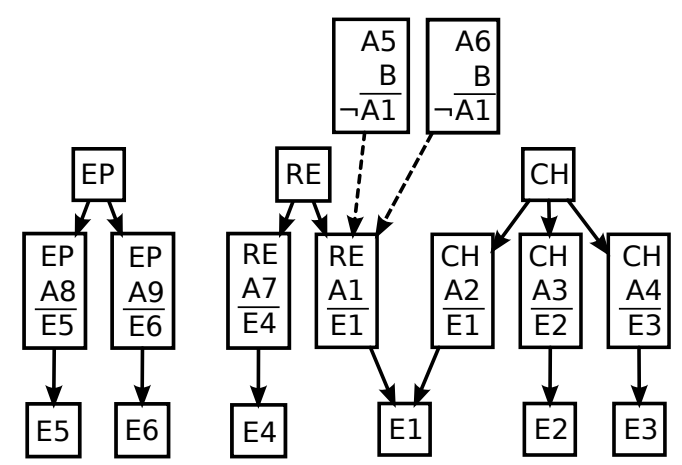

Figure 6: Dialectical structure depicting a stylised reconstruction of the arguments and explanations pertaining to Semmelweis's study of childbed fever. $B$ refers to background knowledge. $A_{1}$ to $A_{9}$ are auxiliary assumptions. See the text for further keys.

\begin{tabular}{|c|c|c|c|c|c|}
\hline & $\operatorname{DoJ}\left(\mathcal{E} \mid \mathcal{H}_{i}\right)$ & $x$ & $\operatorname{Dov}\left(\mathcal{H}_{i}\right)$ & $\propto$ & $\operatorname{DoJ}\left(\mathcal{H}_{i} \mid \mathcal{E}\right)$ \\
\hline $\mathrm{i}=1$ & 0.13 & & 0.21 & & 0.17 \\
\hline$i=2$ & 0.14 & & 0.25 & & 0.23 \\
\hline$i=3$ & 0.30 & & 0.16 & & 0.30 \\
\hline
\end{tabular}

Table 3: Degrees of partial entailment for the reconstruction of Semmelweis's investigation shown in figure 6 .

$$
\begin{aligned}
\mathcal{H}_{3} & :=(\mathrm{CH}) \text { is true } \\
\mathcal{E} & :=(\mathrm{E} 1),(\mathrm{E} 2) \text { and }(\mathrm{E} 3) \text { are true. }
\end{aligned}
$$

As table 3 shows, the cadavric hypothesis $\left(\mathcal{H}_{3}\right)$ implies the total evidence to the greatest degree and displays, in turn, the greatest conditional degree of justification relative to the evidence. Accepting the cadavric hypothesis hence maximizes the robustness of one's position. This coheres with the abductive inference Semmelweis has actually drawn.

\subsection{Meselson's and Stahl's experimental analysis of DNA replica- tion}

In a detailed case study, Weber [2009] gives an account of the experimental study of DNA replication in the 1950s, claiming that inference to the best explanation has figured prominently in this episode of molecular biology. In what follows, I outline Weber's discussion and demonstrate how the pivotal abductive inference he identifies can be justified in the dialectic framework presented above.

After James D. Watson and Francis H. C. Crick unravelled the structure of DNA in the early 1950s, the question arose how, i.e. through which molecular biologic mechanism, DNA molecules are replicated. Soon, three rival hypothesis were 
proposed that described different replication mechanisms.

According to the semi-conservative replication hypothesis (SC), originally suggested by Watson and Crick, the two (identical) strands of a DNA molecule are separated before both strands serve as blueprints to synthesize further, identical strands. Each old strand pairs up with a newly synthesized one to form, finally, a new DNA molecule.

Max Delbrück, doubtful that DNA molecules can be efficiently unravelled, proposed an alternative replication mechanism: so-called dispersive replication (DI). He suggested that DNA strands be synthesized section-wise, whereas newly synthesized and old DNA sections are alternately conjoined to form replicated molecules.

A third mechanism has been described by Gunther Stent. According to the conservative hypothesis (CO), DNA molecules are fully left intact (their two strands are neither separated nor cut and re-conjoined) when being copied.

In 1957, Matthew Meselson and Frank Stahl conducted an experiment in order to investigate DNA replication. They grew microorganisms under different, controlled conditions. Bacteria, when reproducing, use the atomic material available in their environment so as to build new DNA. Meselson and Stahl systematically varied this environment, and the available material that serves as building blocks for new DNA. Specifically, they made two different isotypes of nitrogen (i.e. with different weight) available to the microorganisms, who first grew in an environment with light and then continued to reproduce in an environment with heavy nitrogen. After switching to the new medium with heavy nitrogen, Meselson and Stahl determined the weight of the DNA molecules at regular intervals by using high precision centrifuges. They found DNA molecules whose weight was exactly in between that of DNA which is merely composed of heavy nitrogen and that of DNA which comprises only light nitrogen. Moreover, the weight of DNA molecules didn't vary as the bacteria continued to multiply in the new medium with heavy nitrogen. These data patterns represent the main evidence (E) to be explained.

Weber stresses, in his discussion of the experiment, that the semi-conservative hypothesis yields a straight-forward explanation for this observation. Together with the experimental mechanism (EM), which consists in background knowledge and a key assumption about the functioning of the measurement process $\left(A_{1}\right)$, SC implies the data patterns (E). This is not true for the alternative hypotheses. In contrast, Weber claims, they imply non-E given the experimental set-up. Only by denying the auxiliary assumption $A_{1}$ can the dispersive and the conservative hypothesis be made consistent with the evidence. (This possibility is also the reason why the entire inference does not simply consist in refuting rival hypotheses.) Moreover, only by presuming additional auxiliary assumptions which are not part of the experimental set-up $\left(A_{2}\right.$ respectively $\left.A_{3}\right)$ do DI and CO provide explanations for the evidence. 


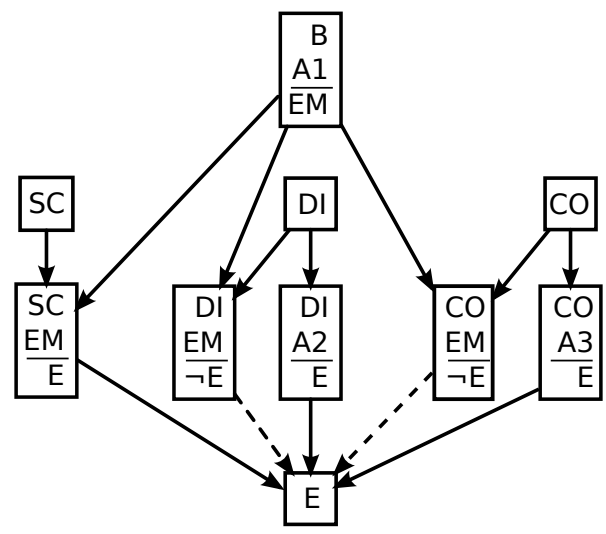

Figure 7: Dialectical structure depicting a stylised reconstruction of the arguments and explanations that underly Meselson's and Stahl's abductive inference concerning DNA replication. $B$ refers to background knowledge. $A_{1}$ to $A_{3}$ are auxiliary assumptions. See the text for further keys.

\begin{tabular}{|c|c|c|c|c|c|}
\hline & $\operatorname{DoJ}\left(\mathcal{E} \mid \mathcal{H}_{i}\right)$ & $\times$ & $\operatorname{DoJ}\left(\mathcal{H}_{i}\right)$ & $\propto$ & $\operatorname{Dou}\left(\mathcal{H}_{i} \mid \mathcal{E}\right)$ \\
\hline$i=1$ & 0.75 & & 0.27 & & 0.38 \\
\hline$i=2$ & 0.4 & & 0.17 & & 0.13 \\
\hline$i=3$ & 0.4 & & 0.17 & & 0.13 \\
\hline
\end{tabular}

Table 4: Degrees of partial entailment for the reconstruction of the experimental investigation into DNA replication shown in figure 7.

This said, Weber argues that the experiment of Meselson and Stahl allows to infer the semi-conservative hypothesis by inference to the best explanation.

Figure 7 presents a dialectical structure which models the argumentative situations faced by Meselson and Stahl (after having carried out the experiments). ${ }^{19}$ The experimental mechanism (EM) is derived from background knowledge and an additional (yet unwarranted) key assumption $A_{1}$. Only SC can explain (derive) E with EM. Its rivals imply non-E, given EM. By assuming further assumptions, DI and CO imply E, as well. We define partial positions as follows.

$$
\begin{aligned}
\mathcal{H}_{1} & :=(\mathrm{SC}) \text { is true } \\
\mathcal{H}_{2} & :=(\mathrm{DI}) \text { is true } \\
\mathcal{H}_{3} & :=(\mathrm{CO}) \text { is true } \\
\mathcal{E} & :=(\mathrm{E}) \text { is true }
\end{aligned}
$$

The degrees of justification of the alternative hypotheses are reported in table 4. The semi-conservative hypothesis SC implies the observed data patterns to the greatest degree $\left(\operatorname{Dov}\left(\mathcal{E} \mid \mathcal{H}_{1}\right)=0.75\right)$. Correspondingly, $\mathrm{SC}$ is itself implied most

\footnotetext{
${ }^{19}$ Again, we assume tacitly that the three hypotheses are contrary.
} 
strongly by the evidence. Hence, assenting to the loveliest explanation, SC, maximizes robustness. This justifies the abductive inference that led to the acceptance of the semi-conservative replication hypothesis in molecular biology.

\subsection{Hume's critique of intelligent design}

The Dialogues Concerning Natural Religion [Hume, 1935] dissect the theory of intelligent design and deliver a trenchant criticism of what Kant later called the teleological proof of the existence of God. As indicated in the introductory section, the teleological proof may be understood as an inference to the best explanation. Hume's critique of this very argument in Part 6 of the Dialogues shall serve as a final test case for our analysis of IBdE.

The Dialogues are, on the whole, concerned with the question how to explain a specific piece of evidence. Namely,

$\left(E_{1}\right)$ All subdivisions of nature are adjusted to each other such that means are adapted to appropriate ends.

In Part 6, particular attention is paid to an additional fact,

$\left(E_{2}\right)$ Nature is a dynamic system characterised by: A continual circulation of matter which produces no disorder; the conservation of matter; the operation of each subdivision so as to preserve both itself as well as the whole.

The hypothesis of intelligent design is put forward, in Part 2, to explain the evidence $E_{1}$.

(ID) Hypothesis of intelligent design: God, i.e. an intelligent, omniscient and almighty being, has created the universe.

Hume seeks to undermine, in Part 6, the (abductive) inference to ID by proposing alternative explanatory hypotheses which explain the available evidence at least as good as ID, if not strictly better. Accordingly, Hume suggests that the evidence $E_{2}$, which is, he contends, only vaguely explained by the assumption that God created the universe, could equally be explained by the hypothesis that God is the soul of the world.

(SW) Hypothesis of Deity as the soul of the world: The universe resembles an animal, and God is its soul, the soul of the world.

The hypotheses ID and SW, excluding each other, represent incompatible rivals. A third explanatory hypothesis, introduced in Part 6, reads,

(IO) Hypothesis of immanent order: Nature possesses an immanent or inherent tendency to self-organisation. 


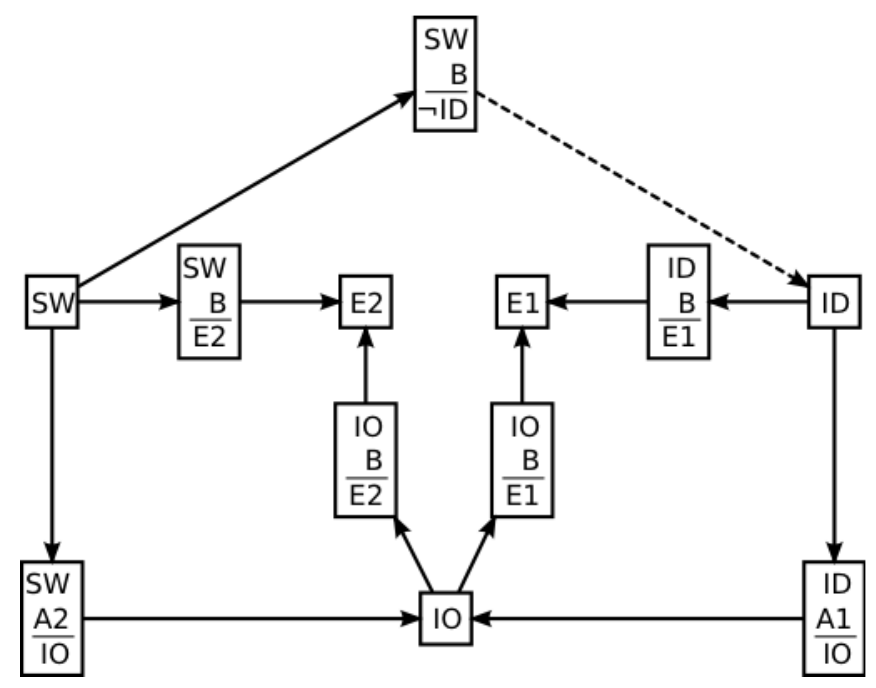

Figure 8: Dialectical structure depicting a stylised reconstruction of Part 6 of Hume's Dialogues. $B$ refers to background knowledge. $A_{1}$ and $A_{2}$ are auxiliary assumptions. See the text for further keys.

Hume claims that IO is sufficiently powerful to explain both pieces of evidence $E_{1}$ and $E_{2}$. On top of that, he maintains that the two hypotheses ID and SW presuppose the existence of some immanent tendency to self-organisation and therefore entail IO anyway. This said, IO has the widest explanatory scope and is prima facie no less plausible than its rivals (for being presupposed by them). Intuitively, IO therefore yields the loveliest explanation of the evidence. Let us see whether this is reflected in an analysis along the lines of this paper. Assuming, for the sake of simplicity, that all the auxiliary assumptions involved in the explanations just sketched belong to the background knowledge, we may reconstruct the dialectical structure as shown in figure $8 .^{20}$ We shall define partial positions as follows.

$$
\begin{aligned}
\mathcal{H}_{1} & :=(\mathrm{ID}) \text { is true, } \\
\mathcal{H}_{2} & :=(\mathrm{SW}) \text { is true, } \\
\mathcal{H}_{3} & :=(\mathrm{IO}) \text { is true }, \\
\mathcal{E} & :=(\mathrm{E} 1) \text { and }(\mathrm{E} 2) \text { are true. }
\end{aligned}
$$

Table 5 displays the resulting degrees of partial entailment. The principle of immanent order (IO) surpasses its rival hypotheses both in terms of degree of partial entailment of the evidence as well as in terms of unconditional degree of justification. Moreover, IO is implied twice as strongly by the evidence as its rivals. Therefore, extending the initial position (B) by IO results in an enlarged position which is

\footnotetext{
${ }^{20} \mathrm{~A}$ much more detailed reconstruction can be found in Betz [2010a, appendix C].
} 


\begin{tabular}{|c|c|c|c|c|c|}
\hline & $\operatorname{DoJ}\left(\mathcal{E} \mid \mathcal{H}_{i}\right)$ & $\times$ & $\operatorname{Dos}\left(\mathcal{H}_{i}\right)$ & $\propto$ & $\operatorname{DoJ}\left(\mathcal{H}_{i} \mid \mathcal{E}\right)$ \\
\hline $\mathrm{i}=1$ & 0.75 & & 0.22 & & 0.3 \\
\hline$i=2$ & 0.75 & & 0.22 & & 0.3 \\
\hline$i=3$ & 1 & & 0.33 & & 0.6 \\
\hline
\end{tabular}

Table 5: Degrees of partial entailment for the reconstruction of Hume's Dialogues shown in figure 8.

more robust than the position augmented by ID or SW. This immediately blocks any inference to the thesis of intelligent design by IBdE. Hume's critique of the teleological argument succeeds. If the evidence $E_{1}$ and $E_{2}$ were to be explained at all - what Hume, by the way, doubts - and one of the alternative explanatory hypotheses should consequently be adopted, if, in other words, the specific instance of the general premiss (G3) were true in this case, then accepting IO, not ID, would be the reasonable thing to do.

\section{Conclusion}

We have argued that IBdEs can be reconstructed as practical meta-syllogisms,

(I1) Accepting the best explanation $H^{*}$ maximises the robustness of one's position relative to accepting any other available explanatory hypothesis.

(I2) It is reasonable to maximise the robustness of one's position.

(I3) One of the explanatory hypotheses should be accepted.

(14) Thus: It is reasonable to accept $H^{*}$.

The first premiss (I1) of such an argument has to be evaluated with respect to a certain state of debate being represented by a dialectical structure. This dialectical structure includes, amongst further arguments, the reconstructed explanations. We have argued, moreover, that the second premiss (I2) holds universally. The third premiss (I3), however, is clearly case sensitive and requires specific attention. If a proponent denies that any explanatory hypothesis should be adopted at all, e.g. because she prefers to remain agnostic and doesn't feel that certain evidence is in urgent need of explanation, she has successfully blocked the IBdE. The metaargument represented by scheme (I) corresponds to the following first order scheme,

(J1) The explanatory hypothesis $H^{*}$ provides, relative to any other available explanatory hypothesis, the best explanation for some evidence $E$.

(J2) $E$ is the case.

(J3) $E$ ought to be explained.

(J4) Thus: $H^{*}$.

Premisses (J1) and (J2) entail premiss (I1) of the meta-argument. Premiss (J3) implies (I3). Thus, we have been able to justify IBdE. In the same time, our analysis has, however, exposed the weak links of IBdE, explaining why this kind of 
reasoning is by and large much less compelling than a sound deductive argument: First of all, it is only reasonable to extend one's position in the most robust way if specific, contestable assumptions are satisfied (cf. J3/I3). In addition, the most robust extension of one's position may still turn out to be false - either because the true hypothesis did not even figure among the alternative explanations, or because an explanatory hypothesis which exhibits a low prima facie plausibility and which hardly explains the evidence turns out to be correct in the long run. In addition to explaining the relative weakness of IBdE, we can account, by reconstructing IBdE as a meta-argument, for IBdE's non-monotonicity. This is because a change of the background knowledge, or the introduction of new arguments alters the dialectical structure with regard to which the premisses of an IBdE are evaluated. As a consequence, the hypothesis that displayed, previously, the highest robustness and that is referred to in premiss (I1) might not do so any more once additional knowledge has been acquired.

I'd like to close this paper with a brief comment on probabilistic explanations. No doubt, statistical explanations seem to defy a deductive reconstruction. If I cite some statistical laws to explain to my nephew why his numbers didn't win the national lottery, my explanation by no means implies deductively that his numbers weren't chosen. ${ }^{21}$ As a consequence, inference to the best statistical explanation (IBsE) cannot be analysed along the lines of this paper. Nevertheless, there seem to be instances of good and successful IBsEs. Just think of medical examples: If the observable symptoms $E$ might by caused by two different diseases while disease 1 typically brings about $E$ whereas disease 2 is rather unlikely to do so, the doctor, or so it seems, rightly infers that the patient suffers from disease 1 , this being the best explanation of the symptoms. Explanatory qualities, even in a statistical setting, seem to guide our inferences quite correctly. Yet, I suspect that such considerations can be fully reconstructed, and thence be justified or corrected, as clear-cut statistical inferences. A theory of statistical reasoning, which may or may not include, for example, the principle of maximum likelihood or Neyman-Pearson testing procedures, is the general framework in which IBsE ought to be studied. I suppose that successful and reliable IBsEs will turn out to be valid statistical arguments.

\section{References}

Gregor Betz. Theorie dialektischer Strukturen. Klostermann, Frankfurt am Main, 2010a.

\footnotetext{
${ }^{21}$ Note that even Railton's deductive-nomological account of probabilistic explanation doesn't allow for reconstructing a statistical explanation as a deductive argument in favour of the explanandum [Railton, 1978].
} 
Gregor Betz. Petitio principii and circular argumentation as seen from a theory of dialectical structures. Synthese, 175(3):327-349, 2010b.

Gregor Betz. On degrees of justification. Erkenntnis, forthcoming, 2011.

Gregor Betz. Debate Dynamics: How Controversy Improves Our Beliefs. Synthese Library. Springer, Dordrecht, forthcoming.

Andrei Bondarenko, Phan Minh Dung, Robert A. Kowalski, and Francesca Toni. An abstract, argumentation-theoretic approach to default reasoning. Artificial Intelligence, 93(1-2):63-101, 1997.

Claudette Cayrol and Marie-Christine Lagasquie-Schiex. On the acceptability of arguments in bipolar argumentation frameworks. In ECSQARU, pages 378-389, 2005.

Phan Minh Dung. On the acceptability of arguments and its fundamental role in nonmonotonic reasoning, logic programming and n-person games. Artificial Intelligence, $77(2): 321-358,1995$.

John Earman. Bayes or Bust? A Critical Examination of Bayesian Confirmation Theory. MIT Press, Cambridge, Mass., 1992.

Carl G. Hempel. Philosophy of Natural Science. Prentice-Hall foundations of philosophy series. Prentice-Hall, Englewood Cliffs, N.J., 1966.

Christopher Hitchcock. The lovely and the probable. Philosophy and Phenomenological Research, LXXIV(2):433-440, 2007.

Colin Howson and Peter Urbach. Scientific Reasoning: The Bayesian Approach. Open Court, Chicago, 3rd edition, 2005.

David Hume. Dialogues Converning Natural Religion. Oxford University Press, Oxford, 1935.

Peter Lipton. Inference to the Best Explanation. Routledge, London, 2004.

John Losee. A Historical Introduction to the Philosophy of Science. Oxford University Press, Oxford, 2001.

Deborah Mayo. Error and the Growth of Experimental Knowledge. Chicago University Press, Chicago, 1996.

Samir Okasha. Van Fraassen's critique of inference to the best explanation. Studies in History and Philosophy of Science, 31A(4):691-710, 2000.

Peter Railton. A deductive-nomological model of probabilistic explanation. Philosophy of Science, 45:206-226, 1978.

Jonah N. Schupbach. Studies in the Logic of Explanatory Power. Dissertation. University of Pittsburgh, 2011.

Jonah N. Schupbach and Jan Sprenger. The logic of explanatory power. Philosophy of Science, 78(1):105-127, 2011. 
Wilfrid Sellars. Are there non-deductive logics? In Nicholas Rescher, editor, Essays in Honour of Carl G. Hempel, pages 83-103. Reidel, Dordrecht, 1969.

Paul Thagard. Computational Philosophy of Science. MIT Press, Cambridge, Mass., 1988.

Paul Thagard. Conceptual Revolutions. Princeton University Press, Princeton, 1992.

Bas C. van Fraassen. Laws and Symmetry. Oxford University Press, Oxford, 1989.

Marcel Weber. The crux of crucial experiments: Duhem's problem and inference to the best explanation. British Journal for the Philosophy of Science, 60:19-49, 2009 .

Bernard Williams. Deciding to believe. In Bernard Williams, editor, Problems of the Self, pages 136-151. Cambridge University Press, Cambridge, 1973. 


\section{General functions}

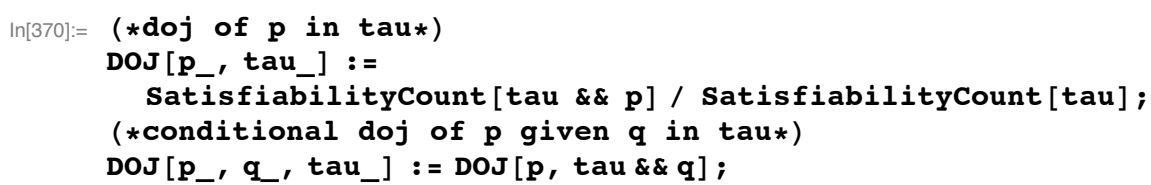

- Calculation of DOJs for Figure 3

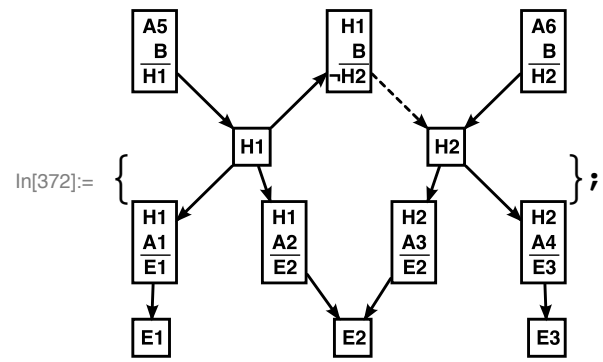

( $*$ Set up the boolean constraints*)

ClearAll [tau, a1, a2, a3, a4, a5, a6, a7, Et, H0, H1, H2] ;

a1 $=($ H1 \&\& B $) \Rightarrow$ ! H2;

a2 $=(A 5 \& \& B) \Rightarrow H 1$;

a3 $=(A 6 \& \& B) \Rightarrow \mathrm{H} 2 ;$

$\mathrm{a} 4=(\mathrm{H} 1 \& \& \mathrm{~A} 1) \Rightarrow \mathrm{E} 1$;

$\mathrm{a} 5=(\mathrm{H} 1 \& \& \mathrm{~A} 2) \Rightarrow \mathrm{E} 2$;

$\mathrm{a} 6=(\mathrm{H} 2 \& \& \mathrm{~A} 3) \Rightarrow \mathrm{E} 2$;

$\mathrm{a} 7=(\mathrm{H} 2 \& \& \mathrm{~A} 4) \Rightarrow \mathrm{E} 3$;

tau $=$ a1 \&\& a2 \&\& a3 \&\& a4 \&\& a5 \&\& a6 \&\& a7;

( $*$ Define total evidence $*$ )

$E t=E 1 \& \& E 2 \& \& E 3$;

( $*$ Define $\mathrm{HO} *$ )

HO $=$ ! H1 \&\& ! H2;

$\ln [384]:=(*$ DOJs as in table $1 *)$

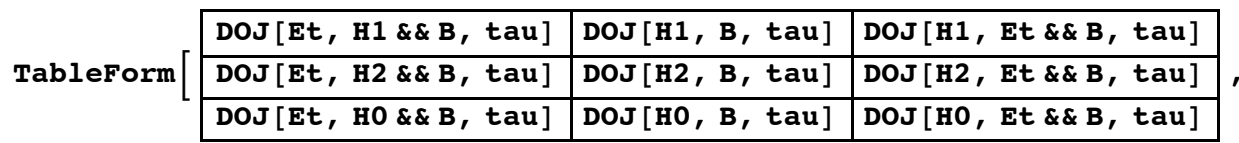

TableHeadings $\rightarrow\{$ None, $\{\mathrm{P}(\mathrm{E} \mid \mathrm{Hi}) ", \mathrm{P}(\mathrm{Hi}) ", \mathrm{P}(\mathrm{E} \mid \mathrm{Hi}) "\}\}]$

Out[384]//TableForm=

\begin{tabular}{lll}
$\mathrm{P}(\mathrm{E} \mid \mathrm{Hi})$ & $\mathrm{P}(\mathrm{Hi})$ & $\mathrm{P}(\mathrm{E} \mid \mathrm{Hi})$ \\
\hline$\frac{2}{9}$ & $\frac{9}{26}$ & $\frac{2}{5}$ \\
$\frac{2}{9}$ & $\frac{9}{26}$ & $\frac{2}{5}$ \\
$\frac{1}{8}$ & $\frac{4}{13}$ & $\frac{1}{5}$
\end{tabular}

In[385]:= TableForm $[N[\%]$, TableHeadings $\rightarrow\{$ None, $\{$ P(E|Hi) ", "P(Hi) ", "P(E|Hi) " $\}$ \}

Out[385]//TableForm=

\begin{tabular}{lll}
$\mathrm{P}(\mathrm{E} \mid \mathrm{Hi})$ & $\mathrm{P}(\mathrm{Hi})$ & $\mathrm{P}(\mathrm{E} \mid \mathrm{Hi})$ \\
\hline 0.222222 & 0.346154 & 0.4 \\
0.222222 & 0.346154 & 0.4 \\
0.125 & 0.307692 & 0.2
\end{tabular}


- Calculation of DOJs for Figure 4

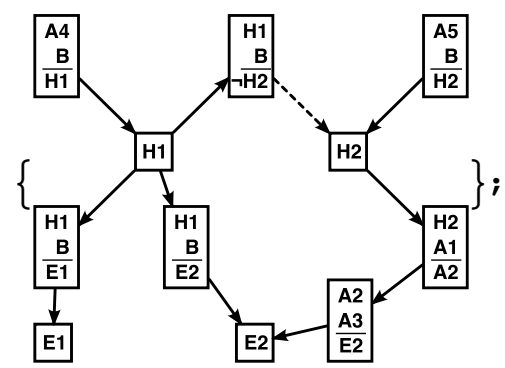

(*Set up the boolean constraints*)

ClearAll[tau, a1, a2, a3, a4, a5, a6, a7, Et, но, н1, н2];

$\mathrm{a} 1=(\mathrm{H} 1 \& \& \mathrm{~B}) \Rightarrow$ ! H2;

$\mathrm{a2}=(\mathrm{A4} \& \& \mathrm{~B}) \Rightarrow \mathrm{H} 1 ;$

a3 $=(A 5 \& \& B) \Rightarrow \mathrm{H} 2$;

$\mathrm{a} 4=(\mathrm{H} 1 \& \& \mathrm{~B}) \Rightarrow \mathrm{E} 1$;

a5 $=(\mathrm{H} 1 \& \& \mathrm{~B}) \Rightarrow \mathrm{E} 2$;

$\mathrm{a} 6=(\mathrm{H} 2 \& \& \mathrm{~A} 1) \Rightarrow \mathrm{A} 2$;

$a 7=(A 2 \& \& A 3) \Rightarrow E 2 ;$

tau $=a 1 \& \&$ a2 \&\& a3 \&\& a4 \&\& a5 \&\& a6 \&\& a7

(*Define total evidence*)

$\mathrm{Et}=\mathrm{E} 1 \& \& \mathrm{E} 2$;

(*Define $\mathrm{HO} *$ )

$\mathrm{HO}=$ ! H1 \&\& ! H2;

(*DOJs as in table $2 *$ )

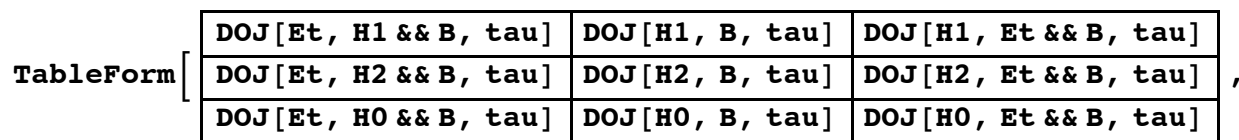

TableHeadings $\rightarrow\{$ None, $\{$ P(E|Hi) ", "P(Hi) ", "P(E|Hi) " $\}\}]$

\begin{tabular}{lll}
$\mathrm{P}(\mathrm{E} \mid \mathrm{Hi})$ & $\mathrm{P}(\mathrm{Hi})$ & $\mathrm{P}(\mathrm{E} \mid \mathrm{Hi})$ \\
\hline 1 & $\frac{4}{21}$ & $\frac{4}{9}$ \\
$\frac{3}{10}$ & $\frac{10}{21}$ & $\frac{1}{3}$ \\
$\frac{2}{7}$ & $\frac{1}{3}$ & $\frac{2}{9}$
\end{tabular}

TableForm $[\mathrm{N}[\%]$, TableHeadings $\rightarrow\{$ None, $\{$ "P (E|Hi) ", "P(Hi) ", "P(Hi|E) "\}\}]

\begin{tabular}{lll}
$\mathrm{P}(\mathrm{E} \mid \mathrm{Hi})$ & $\mathrm{P}(\mathrm{Hi})$ & $\mathrm{P}(\mathrm{Hi} \mid \mathrm{E})$ \\
\hline 1. & 0.190476 & 0.444444
\end{tabular}

$0.3 \quad 0.47619 \quad 0.333333$

$0.285714 \quad 0.333333 \quad 0.222222$ 
- Calculation of DOJs for Figure 5

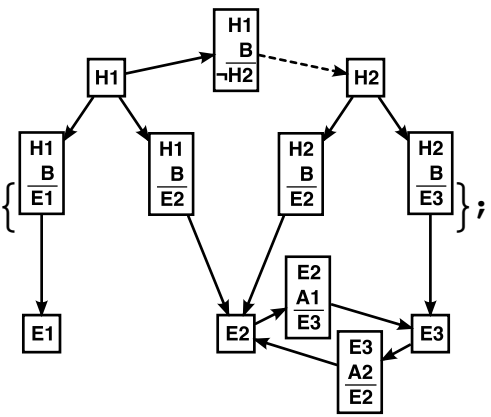

(*Set up the boolean constraints*)

ClearAll [ tau, a1, a2, a3, a4, a5, a6, a7, Et, H0, H1, H2];

$\mathrm{a} 1=(\mathrm{H} 1 \& \& \mathrm{~B}) \Rightarrow$ ! H2 ;

$\mathrm{a} 2=(\mathrm{H} 1 \& \& \mathrm{~B}) \Rightarrow \mathrm{E} 1 ;$

a3 $=(H 1 \& \& B) \Rightarrow E 2 ;$

$\mathrm{a} 4=(\mathrm{H} 2 \& \& B) \Rightarrow E 2 ;$

$\mathrm{a} 5=(\mathrm{H2} \& \& B) \Rightarrow E 3 ;$

$\mathrm{a} 6=(\mathrm{E} 2 \& \& \mathrm{~A} 1) \Rightarrow \mathrm{E} 3$;

a7 $=(\mathrm{E} 3 \& \& \mathrm{A2}) \Rightarrow \mathrm{E} 2$;

tau = a1 \&\& a2 \&\& a3 \&\& a4 \&\& a5 \&\& a6 \&\& a7;

( $*$ Define total evidence $*$ )

$\mathrm{Et}=\mathrm{E} 1 \& \& \mathrm{E} 2 \& \& \mathrm{E} 3$;

(* degree of partial entailment of $E$ by $H 1 *$ )

DOJ $[E t, H 1 \& \& B, t a u]$

2

3

$\mathbf{N}[\%]$

0.666667

(* degree of partial entailment of $\mathrm{E}$ by $\mathrm{H} 2 *$ )

DOJ $[E t, H 2 \& \& B, t a u]$

1

$\overline{2}$

$\mathbf{N}[\%]$

0.5

- Calculation of DOJs for Figure 6

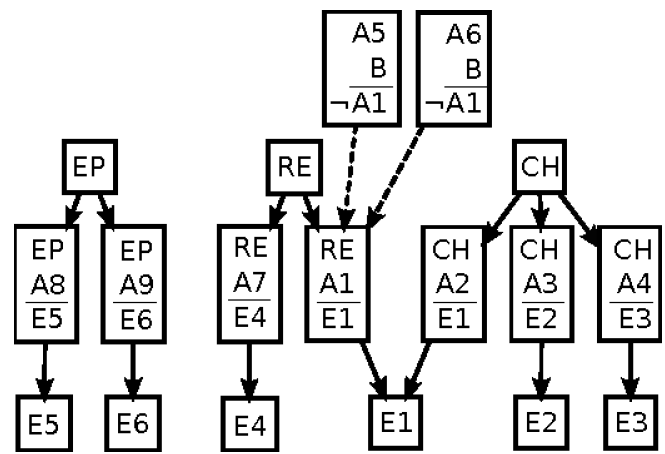




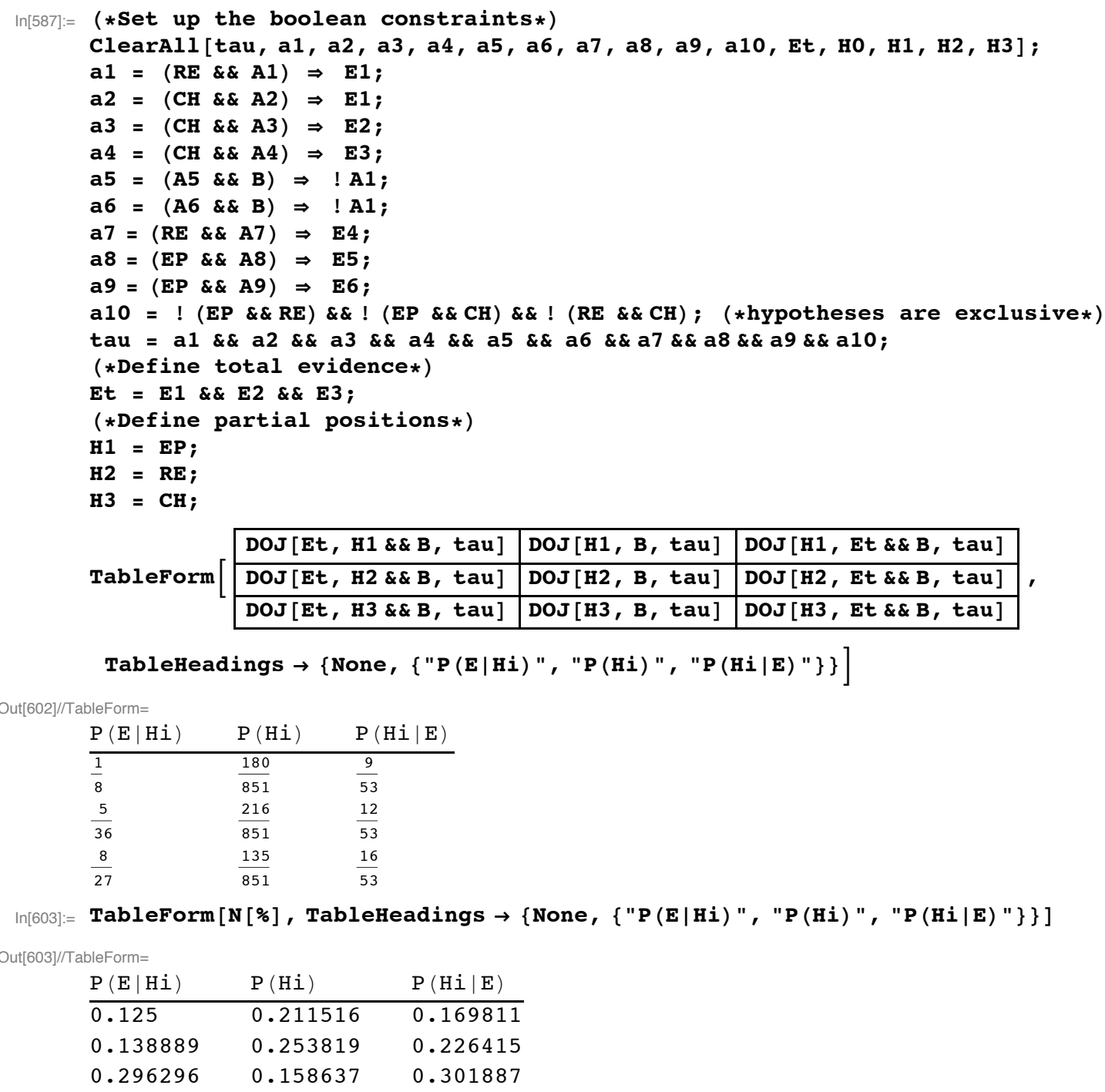

- Calculation of DOJs for Figure 7

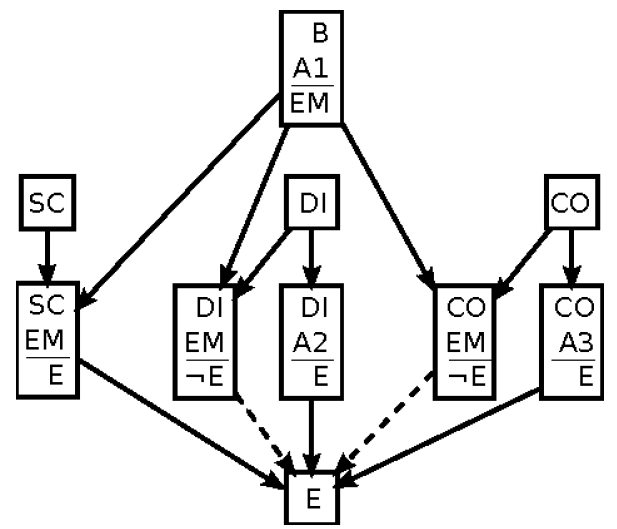


$\ln [604]:=$ (*Set up the boolean constraints*)

ClearAll [tau, a1, a2, a3, a4, a5, a6, a7, a8, a9, a10, Et, H0, H1, H2, H3] ;

$\mathrm{a} 1=(\mathrm{SC} \& \& \mathrm{EM}) \Rightarrow \mathrm{E} 1$;

a2 $=(D I \& \& E M) \Rightarrow ! E 1 ;$

$\mathrm{a} 3=(\mathrm{DI} \& \& \mathrm{A2}) \Rightarrow \mathrm{E} 1 ;$

$\mathrm{a} 4=(\mathrm{CO} \& \& \mathrm{EM}) \Rightarrow \mathrm{I}) \mathrm{E}$;

$\mathrm{a} 5=(\mathrm{CO} \& \& \mathrm{~A} 3) \Rightarrow \mathrm{E} 1$;

$a 6=(A 1 \& \& B) \Rightarrow E M ;$

a7 $=!($ SC \&\&DI) \&\&! (DI \&\&CO) \&\&! (SC\&\&CO); (*hypotheses are exclusive*)

tau $=a 1 \& \& a 2 \& \&$ a3 \&\& a4 \&\& a5 \&\& a6 \&\&a7;

( $*$ Define total evidence*)

Et $=\mathrm{E} 1$;

(*Define partial positions*)

$\mathrm{H} 1=\mathrm{SC}$;

$\mathrm{H} 2$ = DI;

$\mathrm{H} 3=\mathrm{CO}$;

\begin{tabular}{|c|c|c|c|}
\hline \multirow{3}{*}{ TableForm } & DOJ $[E t$, H1 \&\& B, tau ] & $\operatorname{DOJ}[\mathrm{H} 1, \mathrm{~B}, \mathrm{tau}]$ & DOJ $[H 1, E t \& \& B, t a u]$ \\
\hline & DOJ $[E t$, H2 \&\&B, tau] & DOJ $[\mathrm{H} 2, \mathrm{~B}$, tau $]$ & DOJ $[H 2$, Et \&\& B, tau $]$ \\
\hline & DOJ $[E t$, H3 \&\& B, tau] & DOJ $[\mathrm{H3}, \mathrm{B}, \mathrm{tau}]$ & DOJ $[\mathrm{H} 3, \mathrm{Et} \& \& \mathrm{~B}, \mathrm{tau}]$ \\
\hline
\end{tabular}

TableHeadings $\rightarrow\{$ None, $\{$ "P(E|Hi) ", "P(Hi) ", "P(Hi|E) " $\}\}]$

Out[616]//TableForm=

\begin{tabular}{lll}
$\mathrm{P}(\mathrm{E} \mid \mathrm{Hi})$ & $\mathrm{P}(\mathrm{Hi})$ & $\mathrm{P}(\mathrm{Hi} \mid \mathrm{E})$ \\
\hline$\frac{3}{4}$ & $\frac{4}{15}$ & $\frac{3}{8}$ \\
$\frac{2}{5}$ & $\frac{1}{6}$ & $\frac{1}{8}$ \\
$\frac{2}{5}$ & $\frac{1}{6}$ & $\frac{1}{8}$
\end{tabular}

In[617]:= TableForm $[\mathbf{N}[\%]$, TableHeadings $\rightarrow\{$ None, $\{$ "P(E|Hi) ", "P(Hi) ", "P(Hi|E) " $\}\}]$

Out[617]//TableForm=

\begin{tabular}{lll}
$\mathrm{P}(\mathrm{E} \mid \mathrm{Hi})$ & $\mathrm{P}(\mathrm{Hi})$ & $\mathrm{P}(\mathrm{Hi} \mid \mathrm{E})$ \\
\hline 0.75 & 0.266667 & 0.375 \\
0.4 & 0.166667 & 0.125 \\
0.4 & 0.166667 & 0.125
\end{tabular}


- Calculation of DOJs for Figure 8

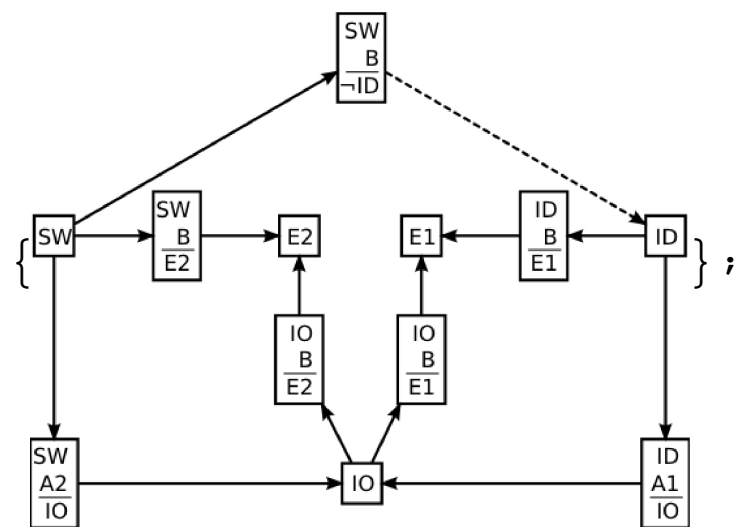

(*Set up the boolean constraints*)

ClearAll[tau, a1, a2, a3, a4, a5, a6, a7, Et, н0, н1, н2, н3] ;

$\mathrm{a} 1=(\mathrm{ID} \& \& \mathrm{~B}) \Rightarrow \mathrm{E} 1$;

$\mathrm{a2}=(\mathrm{SW} \& \& B) \Rightarrow \mathrm{E} 2$;

a3 $=($ SW \&\&B) $\Rightarrow ! I D ;$

$\mathrm{a} 4=(\mathrm{SW} \& \& \mathrm{A2}) \Rightarrow \mathrm{IO}$;

a5 $=($ ID \&\&A1) $\Rightarrow$ IO;

$\mathrm{a} 6=(I O \& \& B) \Rightarrow E 1 ;$

a7 $=($ IO \&\&B $) \Rightarrow E 2 ;$

tau $=a 1 \& \& a 2 \& \& a 3 \& \& a 4 \& \& a 5 \& \& a 6 \& \& a 7 ;$

(*Define total evidence*)

$E t=E 1 \& \& E 2$;

(*Define partial positions*)

H1 = ID;

H2 $=$ SW;

H3 = IO;

(*DOJs as in table $3 *$ )

\begin{tabular}{|c|c|c|c|}
\hline \multirow{3}{*}{ TableForm } & DOJ $[E t$, H1 \&\& B, tau $]$ & DOJ $[\mathrm{H} 1, \mathrm{~B}, \mathrm{tau}]$ & $\operatorname{DOJ}[\mathrm{H} 1, \mathrm{Et} \& \& \mathrm{~B}, \mathrm{tau}]$ \\
\hline & DOJ $[E t, H 2 \& \& B, t a u]$ & DOJ $[\mathrm{H} 2, \mathrm{~B}, \mathrm{tau}]$ & $\operatorname{DOJ}[\mathrm{H2}, \mathrm{Et} \& \& \mathrm{~B}, \mathrm{tau}]$ \\
\hline & DOJ $[E t, H 3 \& \& B, t a u]$ & DOJ $[\mathrm{H} 3, \mathrm{~B}, \mathrm{tau}]$ & $\operatorname{DOJ}[\mathrm{H3}, \mathrm{Et} \& \& \mathrm{~B}, \mathrm{tau}]$ \\
\hline
\end{tabular}

TableHeadings $\rightarrow\{$ None, $\{$ "P(E|Hi) ", "P(Hi) ", "P(Hi|E)" $\}\}]$

\begin{tabular}{lll}
$\mathrm{P}(\mathrm{E} \mid \mathrm{Hi})$ & $\mathrm{P}(\mathrm{Hi})$ & $\mathrm{P}(\mathrm{E} \mid \mathrm{Hi})$ \\
\hline$\frac{3}{4}$ & $\frac{2}{9}$ & $\frac{3}{10}$ \\
$\frac{3}{4}$ & $\frac{2}{9}$ & $\frac{3}{10}$ \\
1 & $\frac{1}{3}$ & $\frac{3}{5}$
\end{tabular}

TableForm $[\mathrm{N}[\%]$, TableHeadings $\rightarrow\{$ None, $\{\mathrm{P}(\mathrm{E} \mid \mathrm{Hi}) ", \mathrm{P}(\mathrm{Hi}) ", " \mathrm{P}(\mathrm{Hi} \mid \mathrm{E}) "\}\}]$

\begin{tabular}{lll}
$\mathrm{P}(\mathrm{E} \mid \mathrm{Hi})$ & $\mathrm{P}(\mathrm{Hi})$ & $\mathrm{P}(\mathrm{Hi} \mid \mathrm{E})$ \\
\hline 0.75 & 0.222222 & 0.3
\end{tabular}

$0.75 \quad 0.222222 \quad 0.3$

1. $\quad 0.333333 \quad 0.6$ 